\title{
Multiobjective Optimal Control of FOPID Controller for Hydraulic Turbine Governing Systems Based on Reinforced Multiobjective Harris Hawks Optimization Coupling with Hybrid Strategies
}

\author{
Wenlong Fu $\mathbb{i D}^{1,2}$ and QiPeng $L u^{1,2}$ \\ ${ }^{1}$ College of Electrical Engineering \& New Energy, China Three Gorges University, Yichang 443002, China \\ ${ }^{2}$ Hubei Provincial Key Laboratory for Operation and Control of Cascaded Hydropower Station, China Three Gorges University, \\ Yichang 443002, China \\ Correspondence should be addressed to Wenlong Fu; ctgu_fuwenlong@126.com
}

Received 16 January 2020; Revised 25 May 2020; Accepted 18 June 2020; Published 18 July 2020

Academic Editor: Saleh Mobayen

Copyright @ 2020 Wenlong Fu and QiPeng Lu. This is an open access article distributed under the Creative Commons Attribution License, which permits unrestricted use, distribution, and reproduction in any medium, provided the original work is properly cited.

\begin{abstract}
The controlling parameter tuning of the hydraulic turbine governing system (HTGS) is always deduced under single operating condition and is not suitable for the changeable operating conditions of the hydraulic turbine. For this purpose, multiobjective optimization problem of fractional order PID (FOPID) controller for HTGS is constructed through the consideration of no-load disturbance and on-load disturbance operation conditions, where the performance indicators of integral time absolute error (ITAE) under both operation conditions are employed as the objective functions. To achieve the optimum, the multiobjective version of newly proposed Harris hawks optimization (MOHHO) is established to solve the optimization issue. Additionally, hybrid strategies which include Latin hypercube sampling initialization, modified differential evolution operator, and mutation operator are coupled into MOHHO (HMOHHO) to promote the global searching capability. Simultaneously, the linear model of rabbit energy within $\mathrm{MOHHO}$ is replaced with a nonlinear one to further enhance the searching capacity. Subsequently, the effectiveness and superiority of the proposed HMOHHO are verified by several multiobjective UF and ZDT test problems. Finally, the practical application and contrastive analysis ascertain that the constructed multiobjective problem of FOPID controller is suitable for HTGS under changeable operating conditions, and the proposed HMOHHO is effective in solving the issue.
\end{abstract}

\section{Introduction}

The Energy Internet [1] is called the core technology of "the third industrial revolution," which aims to replace fossil energy with renewable energy [2] and has attracted sufficient attention worldwide. The Energy Internet heightens the proportion of renewable energy gradually in primary energy production and consumption, thus to establish a sustainable energy supply system. Generally, renewable energy contains regular energy and new energy, where new energy has the characteristics of intermittence, randomness, and poor adjustment ability. By contrast, the main regular energy-hydropower energy possesses the features of low production cost, flexible operation, and no pollution [3]. Besides, with the increasing complexity of energy structure, hydropower undertakes more and more tasks of peak load regulation [4] and frequency modulation [5]. In this situation, the operation safety, reliability, and health management [6] of hydropower generator [7-9] which is the key device during the hydropower energy conversion process are of particular importance.

In the process of frequent working condition transformation, hydraulic turbine governing system (HTGS) acts as the main role for ensuring the effective operation of hydropower generator. Nevertheless, there always exist strong nonlinear characteristics within HTGS, which would affect 
the dynamic performance greatly $[4,10]$. Hence, favourable control strategy is very critical to promote the performance of HTGS. In previous studies, there are various control methods which have been used to solve control problems in HTGS, such as sliding mode control [11-14], fault tolerance control [15], and predictive control [16]. In the controlling field of HTGS, the most common control strategy is traditional PID control strategy which is uncomplicated and is simple to implement [17]. For example, Jiang et al. [18] proposed an optimized PID controller for hydroturbine governing system. Khodabakhshian and Hooshmand [19] proposed a new robust PID controller for automatic generation control of hydroturbine power systems. However, the adjusting ability of traditional PID controller is limited, which would result in poor performance of HTGS under multiple operating conditions. By contrast, the FOPID control strategy can achieve adjustment flexibility for hydropower unit [20], which has been studied by researchers. For example, Wu et al. [21] proposed a fuzzy fractional order PID (FFOPID) controller through the combination of FOPID and fuzzy logic controller. The experimental results showed that the optimized design of FFOPID controller has better control quality than PID, FOPID, and fuzzy PID (FPID) controller. $\mathrm{Xu}$ et al. [22] proposed a robust nonfragile fractional order PID controller to solve pumped turbine unit frequency oscillation along the "S" shape curve under unload operation condition. The simulation experiment verified that the proposed FOPID controller based on the bacterial foraging algorithm had better robustness and practicability than PID controller under no-load operation condition. Li et al. [23] designed a FOPID controller for a pumped storage unit, and results showed that the FOPID controller optimized by gravitational search algorithm combined with Cauchy and Gaussian mutation obtained obvious advantage over other PID controllers.

Apart from the superiority of FOPID controller over PID controller, the parameter tuning of controller is an extremely important step for employment of FOPID controller in HTGS. With the sustainable development of intelligence algorithms in recent years, intelligent algorithms have been widely applied to optimize the control parameters of HTGS by searching the defined available space. The commonly used algorithms include the following: genetic algorithm (GA) [24], particle swarm optimization (PSO) [25, 26], gravitational search algorithm (GSA) [27, 28], ant lion optimization (ALO) [29], sine cosine algorithm (SCA) $[30,31]$, and grey wolf optimization (GWO) [32-34]. Meanwhile, error indicator is always employed to measure the performance when optimizing control parameters with intelligent algorithms. Generally, the parameter selection considering multiple error indexes can make up for the shortcomings of single error index. In particular, with regard to two commonly used indexes, integral squared error (ISE) and integral time squared error (ITSE) which are opposed, system applying ISE index has a response with small overshoot percentage but long settling time, while system applying ITSE index has a response with shorter settling time but without stability margin. In other words, the system designer can set the error index weight percentage based on specific system requirements. For example, Chen et al. [35] proposed chaotic nondominated sorting genetic algorithm II (NSGAII) to optimize a multiobjective optimization problem of FOPID controller's parameter tuning, whose objective functions are composed of ISE and ITSE. Piraisoodi et al. [36] proposed a multiobjective robust fuzzy FOPID controller designed for nonlinear HTGS by using NSGAII. The results showed that the proposed controller had better fitness value and time domain specifications than PID and FOPID controllers, as well as satisfying the conflicting objectives including less settling time and minimum damped oscillations. However, their control parameters are always optimized by intelligent algorithms under a single operating condition, in which case the optimization results may not be adaptive for the changeable operating conditions of hydropower unit.

To promote the feasibility of HTGS under changeable working conditions, it is rather necessary and important to consider the optimal control parameters of HTGS under multiple operating conditions [37]. For example, Zhang et al. [38] proposed an improved NSGAIII algorithm to solve multiobjective FOPID controller optimization problem for pumped turbine governing system (PTGS) under multiworking conditions. Xia et al. [39] proposed a multiobjective PID controller for HTGS based on an improved MOGWO algorithm under multiworking conditions. In this paper, the integral time absolute error (ITAE) indexes of HTGS operating at changeable working conditions are constructed to deduce the optimal control parameters of HTGS under different working conditions. Essentially, the control parameters optimization of HTGS under different working conditions could be summarized as a multiobjective optimization issue, which is expected to be solved by multiobjective optimization algorithms. Some representative multiobjective optimization algorithms include the following: nondominated sorting genetic algorithm III (NSGA-III) [40], multiobjective particle swarm optimization (MOPSO) [41], and multiobjective grey wolf optimizer (MOGWO) [42]. Among the above algorithms, MOPSO and MOGWO are the multiobjective versions of the corresponding single objective algorithms. Inspired by this condition, a multiobjective version of Harris hawks optimization (MOHHO) is structured based on single objective $\mathrm{HHO}$, which is recently proposed by Heidari et al. [43] in 2019 and whose superiority has been ascertained. Furthermore, the multiobjective $\mathrm{HHO}$ is reinforced with hybrid strategies (HMOHHO) including Latin hypercube sampling initialization, modified differential evolution operator, and mutation operator, to deduce the optimal control parameters of FOPID controller for HTGS under multiworking conditions. Simultaneously, the linear model of rabbit energy within $\mathrm{MOHHO}$ is replaced with a nonlinear one to further enhance the searching capacity.

The remainder of this paper is organized as follows: Section 2 briefly introduces the basic concepts of fractional calculus and FOPID controller. In Section 3, HTGS model and its control issues are discussed. The proposed $\mathrm{HMOHHO}$ algorithm is constructed in Section 4. In Section 5 , the proposed HMOHHO is compared with NSGAIII, 
MOPSO, MOGWO, and MOHHO and performance analysis is accomplished. In Section 6, comparison of control effects between PID and FOPID controllers is described. Then the Pareto-optimal sets with different algorithms are compared and analyzed, and the transient process responses with different control schemes are presented. Lastly, Section 7 summarizes the conclusions.

\section{Fractional Calculus and the Fractional Order PID}

2.1. Theory of Fractional Calculus. The research of fractional calculus [44] has been carried out since 1960s and widely expanded into the fields of science and technology. During the practical applications, there are always some complex issues which make it difficult to explain the model with integer order calculus, while fractional calculus shows more flexibility and availability. For this purpose, the theory of fractional calculus is introduced and then employed to promote the controller modeling for HTGS later. Here, a unified fractional calculus operator $\left(t_{0} D_{t}^{\alpha}\right)$ is introduced and defined as

$$
t_{0} D_{t}^{\alpha} f(t)= \begin{cases}\frac{\mathrm{d}^{\alpha}}{\mathrm{d} t^{\alpha}} f(t), & \alpha>0, \\ f(t), & \alpha=0, \\ \int_{t_{0}}^{t} f(\tau)(\mathrm{d} \tau)^{-\alpha}, & \alpha<0,\end{cases}
$$

where $\alpha$ is limited to real number, $t$ is an independent variable, and $t_{0}$ is the lower boundary of $t$.

The definition of fractional calculus was firstly proposed in 1868 , which meant the real establishment in the field of fractional calculus. Compared with the definitions of Riemann-Liouville and Grünwald-Letnikov [45], the definition proposed by Caputo [46] is more suitable for conditions with nonzero initialization. The function of fractional derivative defined by Caputo is

$$
{ }_{t_{0}}^{c} D_{t}^{\alpha} y(t)=\frac{1}{\Gamma(m-\alpha)} \int_{t_{0}}^{t} \frac{y^{m}(\tau)}{(t-\tau)^{1+\alpha-m}} \mathrm{~d} \tau,
$$

where $\alpha \in R, m \in Z, m-1<\alpha \leq m, \gamma$ is limited to real number, and $\Gamma(\cdot)$ is Euler Gamma function.

As it can be seen from formula (2), Caputo's definition requires that the $m$-th derivatives of the function are integrable [47]. The Laplace transform of fractional derivative defined by Caputo is expressed in

$$
\int_{0}^{\infty} e^{-s t} D^{\gamma} f(t) \mathrm{d} t=s^{\gamma} F(s)-\sum_{k=0}^{n-1} s^{\gamma-k-1} f^{(k)}(0),
$$

where $n$ is the smallest integer, $\gamma$ donates the order of fractional derivative, and $n-1<\gamma \leq n$.

On account of unappealing outcomes for frequency response fitting with design method of filter based on continued fraction, the filter of fractional order operator proposed by Oustaloup can choose the frequency bands and order, which can approximate the fractional calculus operator with integer order transfer function. However, approximation effect of Oustaloup filter at selected frequency bands boundary is unsatisfactory. This article uses an improved filter form Oustaloup filter [47], whose mathematical model is

$$
s^{\gamma} \approx\left(\frac{\mathrm{d} w_{\mathrm{h}}}{b}\right)^{\gamma}\left(\frac{\mathrm{d} s^{2}+b \omega_{\mathrm{h}} s}{\mathrm{~d}(1-\gamma) s^{2}+v w_{\mathrm{h}} s+\mathrm{d} \gamma}\right) \prod_{k=1}^{N} \frac{s+\omega_{k}^{\prime}}{s+\omega_{k}} .
$$

In formula (4), the zero point, pole point, and gain can be calculated, respectively, as follows:

$$
\begin{aligned}
& \omega_{\mathrm{u}}=\sqrt{\frac{\omega_{\mathrm{h}}}{\omega_{\mathrm{b}}}}, \\
& \omega_{k}^{\prime}=\omega_{\mathrm{b}} \omega_{\mathrm{u}}^{(2 k-1-\gamma) / N}, \\
& \omega_{k}=\omega_{\mathrm{b}} \omega_{\mathrm{u}}^{(2 k-1-\gamma) / N}, \\
& K=\omega_{\mathrm{h}}^{\gamma},
\end{aligned}
$$

where $\left(\omega_{b}, \omega_{h}\right)$ denotes frequency band, $\gamma \in(0,1)$. In general, the weighting parameter is selected as $b=10$ and $d=9$. The filter order $N$ is set at 13 and the frequency band is set $\left(10^{-5}\right.$, $10^{3}$ ) in this paper.

2.2. Basic Concepts of FOPID Controller. The parameters $\lambda$ and $\mu$ of FOPID controller can be set any real number between 0 and 2, which is a generalized form of traditional integer order PID controller. Compared with the traditional integer order PID controller, the parameter tuning of FOPID controller increases the complexity of algorithmic calculation process to a certain extent due to the additional two parameters. However, FOPID controller plays a great important role in improving the flexibility, robustness, and overall control effect of system.

The mathematical models of FOPID controller including the time-domain model and frequency-domain transfer function model are

$$
\begin{aligned}
u(t) & =K_{\mathrm{P}} e(t)+K_{\mathrm{i}} D^{-\lambda} e(t)+K_{\mathrm{d}} D^{\mu} e(t), \\
C(s) & =K_{\mathrm{p}}+K_{\mathrm{i}} s^{-\lambda}+K_{\mathrm{d}} s^{\mu}, \\
\lambda, \mu & >0,
\end{aligned}
$$

where $\lambda=1$ and $\mu=1$; it is the traditional PID controller model.

\section{Multiobjective Optimization Framework for FOPID and PID Controllers in HTGS}

3.1. HTGS Model. HTGS system, including governor, hydraulic servo system, hydraulic turbine, penstock, and generator [48], is influenced by hydraulic, mechanical, and electrical factors, making its response behavior complicated. The structure diagram of HTGS is shown in Figure 1. As far as system modeling is concerned, the simulation system expressed roundly by various factors plays a significant role in promoting the reliability of simulation results. Nevertheless, in the modeling of actual complex systems, there 
always exist many influencing factors which are difficult to be considered totally, which means that the least important factors are neglected in most cases.

During the transition process of hydropower station, the change of flowing water in the penstock would induce water hammer effect. When the length of penstock is less than 800 meters, it is considered that the elasticity of water body and penstock wall has little effect on the water hammer, amounting to that water hammer pressure spreading to the entire penstock is accomplished instantaneously. The rigid water hammer model of water diversion system is

$$
G_{\mathrm{h}}(s)=\frac{h(s)}{q(s)}=-T_{\mathrm{w}} s,
$$

where $T_{\mathrm{w}}$ is the water flow inertia time constant, which is an important parameter in the water diversion system.

Torque and flow rate of the Francis hydraulic turbine are related to the guide vane opening, rotational speed, and water head. The Francis hydraulic turbine model in steady state can be expressed as

$$
\left\{\begin{array}{l}
\mathrm{mt}=\mathrm{mt}(y, h, \omega), \\
q=q(y, h, \omega),
\end{array}\right.
$$

where $m_{\mathrm{t}}, q, \omega, y$, and $h$, respectively, represent relative torque deviation relative value, flow deviation relative value, rotational speed deviation relative value, guide vane opening deviation relative value, and head deviation relative value.

The Taylor expansion of equation (8) is simplified by omitting the second order and higher order differential components [49]. Thus, the following formula can be obtained:

$$
\begin{aligned}
\mathrm{mt} & =e_{\mathrm{x}} \omega+e_{\mathrm{y}} y+e_{\mathrm{h}} h, \\
q & =e_{\mathrm{qx}} \omega+e_{\mathrm{qy}} y+e_{\mathrm{qh}} h,
\end{aligned}
$$

where $e_{\mathrm{x}}$ denotes the first order partial derivative value of torque in relation to speed of hydraulic turbine, $e_{\mathrm{y}}$ denotes first order partial derivative value of torque in relation to wicket gate, $e_{\mathrm{h}}$ denotes the first order partial derivative value of torque with respect to water head, $e_{\mathrm{qx}}$ denotes the first order partial derivative value of flow rate in relation to speed of hydraulic turbine, $e_{\mathrm{qy}}$ denotes the first order partial derivative value of flow rate in relation to wicket gate, and $e_{\mathrm{qh}}$ denotes the first order partial derivative value of flow rate in relation to water head.

The generator is also a complicated subsystem, which is divided into different models according to the order of differential equation. In this paper, the first order generator model is introduced and researched:

$$
G_{\mathrm{g}}(s)=\frac{x(s)}{\mathrm{mt}(s)-\mathrm{mg}(s)}=\frac{1}{T_{\mathrm{a}} s+e_{\mathrm{g}}},
$$

where $x$ donates the frequency of generator, mg represents load torque relative deviation, $T_{\mathrm{a}}$ represents generator mechanical time, and $e_{\mathrm{g}}$ represents generator load self-regulation parameters.
Dead zones and amplitude limit units have been considered in hydraulic servo subsystem. The transfer function of the hydraulic actuator can be expressed as

$$
G_{\mathrm{y}}(s)=\frac{1}{T_{\mathrm{y}} s+1} .
$$

where $T_{\mathrm{y}}$ is the major relay connecter response time.

According to the mathematical model described above, the system block diagram of hydraulic turbine unit can be modeled as shown in Figure 2.

The governor is the key equipment for automation of hydropower station. In other words, the control strategy directly affects the safety and stability of hydropower station and unit. The block diagrams of PID and FOPID controller (the amplitude limit unit is ignored) are shown in Figure 3.

\subsection{Multiobjective Optimal Control of HTGS and Problem} Description. During the process of generating electricity in hydropower station, speed control of HTGS is an extremely important link of the unit control and automation, the quality of which is affected by the parameter tuning of controller. In essence, the parameter selection of controller is an optimization problem to solve extreme values. According to the system requirements, objective function is set with the error performance indicator firstly. Then, a certain method is used to optimize the parameters of controller by objective function. The most commonly used performance indicators $[35,50]$ are ISE, integral absolute error (IAE), ITSE, ITAE, integral squared time squared error (ISTSE), and integral squared time absolute error (ISTAE).

The dynamic responding performance of HTGS is vital for the robustness and stability of power system, which is influenced by load fluctuation and severe frequency interference. Thus, it is expected that the performance objective functions can achieve accurate and robust tracking control. In this paper, ITAE which is one of the most widely used indicators with stable regulation and small overshoot is chosen as the objective function under multiple operating conditions to obtain better transient dynamic performance of system. The ITAE index [35] is defined as follows:

$$
\operatorname{ITAE}=\int_{0}^{T} t|e(t)| \mathrm{d} t,
$$

where $e(t)$ denotes the relative deviation of the rotational speed within HTGS.

Considering two classic operating conditions, single objective HHO is applied to optimize parameters of FOPID controller under $4 \%$ step disturbance condition (no-load operation condition) with the transfer parameters in Table 1 and then HTGS is controlled with the obtained parameters under $4 \%$ load shedding condition (on-load operation condition). The parameters are tuned as $K_{\mathrm{p}}=3.5831$, $K_{\mathrm{i}}=1.1739, K_{\mathrm{d}}=4.5975, \lambda=1.0009$, and $\mu=0.0010$. Besides, the control effects of tuned parameters under two operating conditions are shown in Figure 4, from which it can be seen that the parameters of FOPID controller obtained under noload disturbance are not suitable for the on-load disturbance. In other words, the parameters of controller obtained 


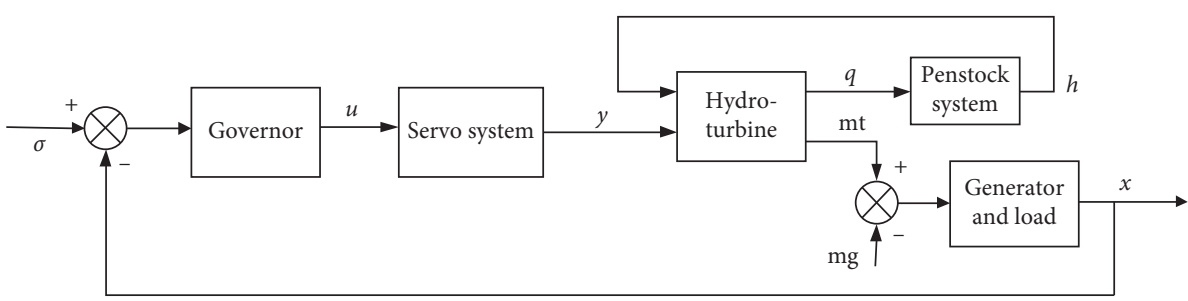

FIGURE 1: The structure diagram of HTGS.

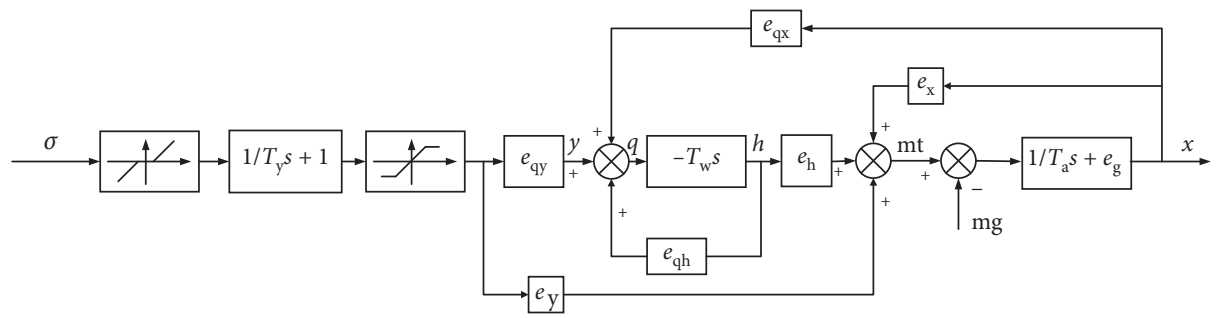

Figure 2: The system block diagram of hydraulic turbine unit.

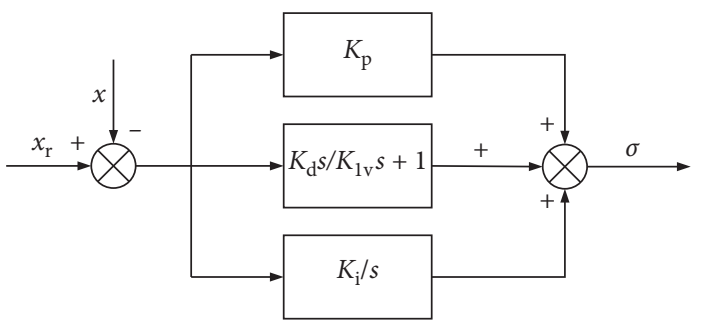

(a)

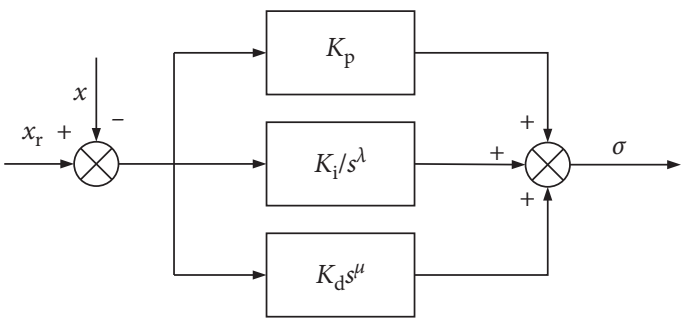

(b)

FIgURE 1: Controllers: (a) PID and (b) FOPID.

TABLE 1: Transferring parameters in hydroturbine and generator under two working conditions.

\begin{tabular}{lccccccccccc}
\hline Running condition & $e_{\mathrm{x}}$ & $e_{\mathrm{y}}$ & $e_{\mathrm{h}}$ & $e_{\mathrm{qx}}$ & $e_{\mathrm{qy}}$ & $e_{\mathrm{qh}}$ & $e_{\mathrm{g}}$ & $T_{\mathrm{a}}$ & $T_{\mathrm{w}}$ & $T_{\mathrm{y}}$ & $K_{1 \mathrm{v}}$ \\
\hline On-load & -0.312 & 1.112 & 0.468 & 0.356 & -0.402 & 0.301 & 0.89 & 8 & 0.56 & 0.1 & 0.28 \\
No-load & -1.004 & 1.341 & 1.293 & 0.369 & 1.074 & 0.297 & 0.89 & 8 & 0.56 & 0.1 & 0.28 \\
\hline
\end{tabular}

under single operating condition are not desirable for the control of hydraulic turbine unit under variable operating conditions.

The main reason of conclusion above is that no-load disturbance and on-load disturbance operation condition are extreme operating conditions in operating process of hydraulic turbine unit. Therefore, optimal control of HTGS is essentially a multiobjective optimization problem. Different operating conditions are considered in this paper for optimal control. Referring to [38], the objective functions of HTGS based on ITAE under two conditions are described as follows:

$$
\begin{aligned}
& \min \left\{\begin{array}{l}
f_{1}=\operatorname{ITAE}_{1}=f_{1}\left(K_{\mathrm{p}}, K_{\mathrm{i}}, K_{\mathrm{d}}, \lambda, \mu\right) \\
f_{2}=\mathrm{ITAE}_{2}=f_{2}\left(K_{\mathrm{p}}, K_{\mathrm{i}}, K_{\mathrm{d}}, \lambda, \mu\right)
\end{array}\right. \\
& \text { subject to }\left\{\begin{array}{l}
K_{\mathrm{p} \text { min }} \leq K_{\mathrm{p}} \leq K_{p \max } \\
K_{\mathrm{i} \text { min }} \leq K_{\mathrm{i}} \leq K_{i \max } \\
K_{\mathrm{d} \min } \leq K_{\mathrm{d}} \leq K_{d \max } \\
\lambda_{\min } \leq \lambda \leq \lambda_{\max } \\
\mu_{\min } \leq \mu \leq \mu_{\max }
\end{array}\right.
\end{aligned}
$$




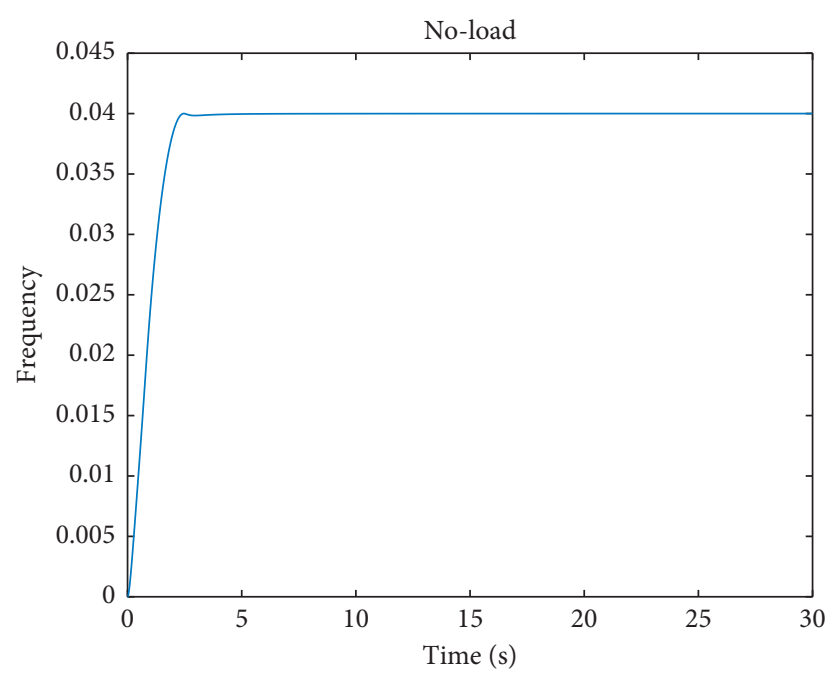

(a)

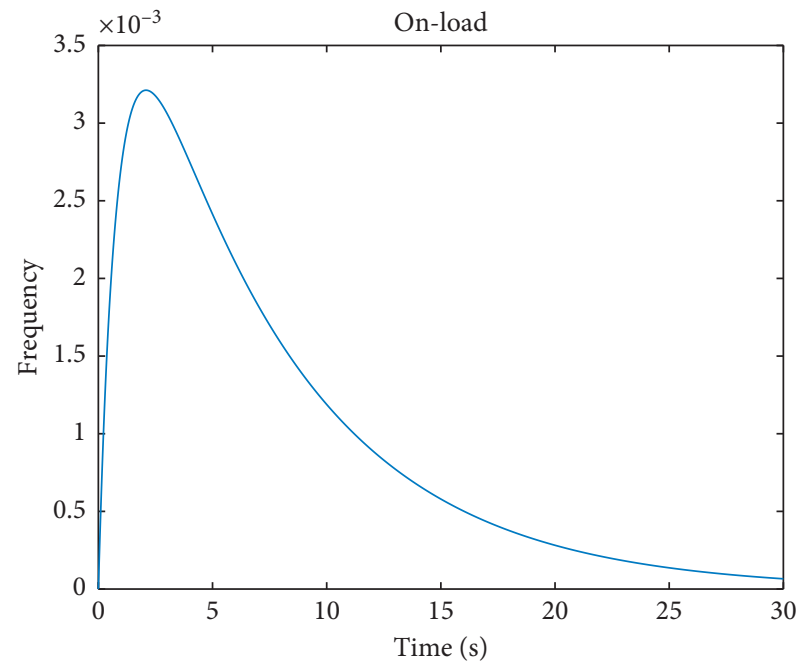

(b)

FIgURE 4: Control effects of the same parameter under two operating conditions. (a) No-load operating condition; (b) on-load operating condition.

where $f_{1}(\cdot)$ and $f_{2}(\cdot)$ are functions of $K_{\mathrm{p}}, K_{\mathrm{i}}, K_{\mathrm{d}}, \lambda$, and $\mu$ under on-load and no-load disturbance running conditions; the lower and upper bounds of $K_{\mathrm{p}}, K_{\mathrm{i}}, K_{\mathrm{d}}, \lambda$, and $\mu$ are $K_{\mathrm{pmin}}=0$, $K_{\text {pmax }}=10, \quad K_{\text {imin }}=0, \quad K_{\text {imax }}=10, \quad K_{\text {dmin }}=0, \quad K_{\text {dmax }}=10$, $\lambda_{\min }=0, \lambda_{\max }=2, \mu_{\min }=0$, and $\mu_{\max }=2$.

\section{Reinforced Multiobjective Harris Hawks Optimization with Hybrid Strategies}

4.1. Harris Hawks Optimization. Mainly inspired by the chasing style and cooperative behavior of Harris hawks,
Heidari and his team developed HHO algorithm which has two main stages: exploration stage and exploitation stage [43]. During exploration stage, Harris's hawks inhabit in random places, waiting to detect prey based on two strategies. The mathematical model is as follows:

$$
X(t+1)= \begin{cases}X_{\text {rand }}(t)-r_{1}\left|X_{\text {rand }}(t)-2 r_{2} X_{t}\right|, & q \geq 0.5, \\ \left(X_{\text {rabbit }}(t)-X_{\mathrm{m}}(t)\right)-r_{3}\left(\mathrm{LB}+r_{4}(\mathrm{UB}-\mathrm{LB})\right), & q<0.5,\end{cases}
$$

where $X(t+1)$ represents the position of hawks in $t+1$ th iteration, $X_{\text {rand }}(t)$ donates the position of hawks chosen randomly in the current population, $r_{1}, r_{2}, r_{3}, r_{4}$, and $q$ are all random numbers in scope of $(0,1), X_{\text {rabbit }}(t)$ is the position vector of rabbit, $X_{t}$ represents the current position of hawk, which is updated during each iteration, LB and UB denote the upper and lower limits of variable, and $X_{\mathrm{m}}(t)$ represents the average position of hawks in the current population, which can be calculated as

$$
X_{\mathrm{m}}(t)=\frac{1}{N} \sum_{i=1}^{N} X_{i}(t)
$$

where $X_{i}(t)$ denotes the location of each hawk in $t$-th iteration and $N$ represents the amount of hawks.

During the transition phase from exploration to exploitation, the energy of rabbit is modeled as

$$
E=2 E_{0}\left(1-\frac{t}{T}\right)
$$

where $E$ is the evasion energy of rabbit, $T$ denotes the maximum iterations, and $E_{0}$ is a value in scope of $(-1,1)$, indicating the initial energy of each step.

When $E>1$, optimization process of Harris hawks is focused mainly in the exploration stage; otherwise, it turns to the exploitation stage which includes the stages of soft besiege, hard besiege, soft besiege with progressive rapid dives, and hard besiege with progressive rapid dives.

In soft besiege stage $(r \geq 0.5$ and $|E| \geq 0.5)$, the behavioral model can be constructed as follows:

$$
\begin{array}{r}
X(t+1)=\Delta X(t)-E\left|J X_{\text {rabbit }}(t)-X(t)\right|, \\
\Delta X(t)=X_{\text {rabbit }}(t)-X(t),
\end{array}
$$


where $\Delta X(t)$ is the difference value between the position of rabbit and the current position in iteration $t, J=2\left(1-r_{5}\right)$ denotes the random energy of rabbit during the escaping procedure, and $r_{5}$ is the random number in scope of $(0,1)$. The changed value of $J$ is realized to simulate the trait of rabbit's escaping movement.

In hard besiege stage $(r \geq 0.5$ and $|E| \leq 0.5)$, the current position is updated by the following formula:

$$
X(t+1)=X_{\text {rabbit }}(t)-E|\triangle X(t)| .
$$

In soft besiege with progressive rapid dives stage $(r<0.5$ and $|E| \geq 0.5)$, in order to carry out advanced soft besiege, hawks can assess their next action according to the following rule:

$$
Y=X_{\text {rabbit }}(t)-E\left|J X_{\text {rabbit }}(t)-X(t)\right| .
$$

The next step will also use the following rule for diving based on levy flight (LF) mode:

$$
Z=Y+S \times \operatorname{LF}(D),
$$

where $D$ represents the dimension of decision vector, $S$ represents a $1 \times D$ random vector in scope of $(-1,1)$, and $L F$ shows the levy flight function formulated as

$$
\begin{aligned}
\operatorname{LF}(x) & =0.01 \times \frac{u \times \sigma}{|v|^{1 / \beta}}, \\
\sigma & =\left(\frac{\Gamma(1+\beta) \times \sin (\pi \beta / 2)}{\Gamma((1+\beta) / 2) \times \beta \times 2^{(\beta-1) / 2}}\right)^{1 / \beta}, \\
\beta & =1.5,
\end{aligned}
$$

where $u$ and $v$ are both random values in scope of $(0,1)$.

Therefore, in order to get a better position vector, the last strategy of soft besiege with progressive rapid dives stage can be expressed as

$$
X(t+1)= \begin{cases}Y, & \text { if } F(Y)<F(Z) \\ Z, & \text { if } F(Z)<F(Y) .\end{cases}
$$

In hard besiege with progressive rapid dives stage $(r<0.5$ and $|E|<0.5)$, the following rules are executed in hard besiege condition:

$$
X(t+1)= \begin{cases}Y, & \text { if } F(Y)<F(Z) \\ Z, & \text { if } F(Z)<F(Y),\end{cases}
$$

where $Y$ and $Z$ can be deduced by the following novel rules:

$$
\begin{array}{r}
Y=X_{\text {rabbit }}(t)-E\left|J X_{\text {rabbit }}(t)-X_{\mathrm{m}}(t)\right|, \\
Z=Y+S \times \operatorname{LF}(D) .
\end{array}
$$

\subsection{Reinforced MOHHO with Hybrid Strategies}

4.2.1. MOHHO. Multiobjective Harris hawks optimization algorithm (MOHHO) based on $\mathrm{HHO}$ is expected to solve multiobjective optimization problem. The steps of $\mathrm{MOHHO}$ algorithm are as follows:
Step 1: preset the population size $N$, maximum iterations $T$, and archive size $S$ at the beginning as well as initializing the population randomly within the given ranges of variables

Step 2: calculate the objective values $f_{1}(\cdot)$ and $f_{2}(\cdot)$ for each hawk $X(t)$

Step 3: select the nondominated Pareto-optimal solutions in the population at the current iteration; thus, the position of rabbit $X_{\text {rabbit }}(t)$ is acquired by leader selection mechanism, whose role is taking advantage of crowding distance to select a solution through roulette wheel method from a less populated area of the archive Step 4: update each hawk $X(t)$ according to equations (14) $-(24)$

Step 5: calculate the new objective value for each hawk and find the nondominated solutions; thus better solutions will be recorded in the archive

Step 7: when the archive is full, the crowded area of the archive is deleted by the roulette wheel method for adding new solutions to the archive

Step 8: output the archive solutions

4.2.2. Reinforced MOHHO. Previous literatures [51-54] have shown that heuristic search algorithms generally suffer from the problem of trapping in local optimum easily, leading to the loss of solution diversity. Similarly, the original iterative process of MOHHO is not sufficient to maintain lateral diversity and achieve a Pareto front of good astringency and high diversity. In order to promote the searching capacity of MOHHO, the linear model of rabbit energy is replaced with a nonlinear one, as shown in

$$
E=2 E_{0}\left(1-\left(\frac{t}{T}\right)^{6}\right) .
$$

In addition to the improvement above, hybrid strategies are merged into $\mathrm{MOHHO}$ to jump out local optimum and search for more no-domain solutions. Firstly, population initialization is conducted through Latin hypercube sampling, which is the latest development in sampling technology [55]. The steps of Latin hypercube sampling initialization are as follows: (1) divide each dimension into $N$ intervals that do not overlap each other; (2) randomly select a point within each interval for each dimension; (3) combine them into a vector. The mathematical model can be described by the following formula:

$$
x_{d_{-} \min }=x_{d}^{0}<x_{d}^{1}<x_{d}^{2}<\cdots<x_{d}^{j}<\cdots<x_{d}^{N}=x_{d_{-} \max },
$$

where $x_{d}$ donates the $d$-th dimension of each hawk vector and $N$ donates population size.

The modified differential evolution operator of the proposed hybrid strategies is shown in

$$
X(t+1)=X_{\text {rand (1) }}+F *\left(X_{\text {rand (2) }}-X_{\text {rand (3) }}\right),
$$


where $X_{\text {rand(1) }}, X_{\text {rand(2) }}$, and $X_{\text {rand(3) }}$ are the positions of hawks chosen randomly in the current population, $F$ is a $1 \times$ dim vector whose value intervals for all dimensions are 0.3 and 0.8 , and dim is the dimension number of each hawk.

The mutation operator mathematical model of the proposed hybrid strategies can be expressed as

$$
\begin{aligned}
d & =m *(\operatorname{UB}(j)-\operatorname{LB}(j)), \\
j & \in \operatorname{rand}\{1,2, \ldots, \operatorname{dim}\}, \\
X(t+1) & =X(j)=X(j)+d,
\end{aligned}
$$

where $m$ is a random value in scope of $(-0.5,0.5)$.

The specific pseudocode of the proposed $\mathrm{HMOHHO}$ algorithm is depicted in Algorithm 1.

4.3. Computational Complexity Analysis. The complexity analysis of each iteration in the proposed $\mathrm{HMOHHO}$ is as follows: assume that individual number is $N$ and the dimension of each hawk is dim, and the number of individuals in the current archive set is assumed to be A. In $\mathrm{HMOHHO}$, the rabbit position in archive set is selected by leader selection mechanism, during which process the computational complexity can be denoted by $O(A)$. Then, computational complexity of location updating can be denoted by $O(N * \operatorname{dim})$. Additionally, the computational complexity of the proposed hybrid strategies can be denoted by $O(2 * N * \operatorname{dim})$ and the computational complexity of updating archive set can be denoted by $O(A * N * \operatorname{dim})$. Next, the computational complexity of grouping and sorting archive sets is $O\left(A^{2}\right)$. On the whole, the computational complexity of each iteration in $\mathrm{HMOHHO}$ is $O\left(A^{2}\right)+O(A * N * \operatorname{dim})$.

\section{Algorithm Performance Verification}

5.1. Verification Experiment Design. In order to verify the availability of the proposed $\mathrm{HMOHHO}$ algorithm, four multimode UF test functions and three ZDT test functions $[56,57]$ are employed to prove the performance. The test functions are shown in Table 2. Besides, NSGA-III, MOPSO, MOGWO, and MOHHO algorithms are introduced for comparison.

5.2. Parameter Setting. The principle and implementation of different algorithms are not uniform exactly. Consequently, relatively fair comparisons of different algorithms are achieved by setting the same maximum iterations, individual dimensions of population, population size, and archive size. The above four parameters of all algorithms for UF problems are set to $1000,30,100$, and 100 severally. For problems ZDT1 and ZDT3, the parameters are set to 200, 30, 100, and 100 severally. For problem ZDT6, the parameters are set to 200, 10, 100, and 100, respectively. In MOGWO and MOPSO, the values of inflation rate, leader selection pressure, deletion selection pressure, and number of grids per dimension are both set to $0.1,4,2$, and 10 , respectively. The values of inertia, inertia weight damping rate, and mutation rate are set to $0.5,0.99$, and 0.1 in MOPSO, respectively. The values of crossover rate, number of neighbors, and mutation rate in NSGAIII are set to $0.5,10$, and 0.1 , respectively.

5.3. Assessment Metrics. To evaluate the algorithm performance, there exist a number of assessment metrics, such as generational distance (GD), spacing (SP), inverted generational distance (IGD), maximum spread (MS), hypervolume $(\mathrm{HV})$, and diversity metric $(\Delta)$ [58]. Among the previous metrics, IGD and HV which can measure convergence and diversity of solutions are chosen to evaluate the performance of each algorithm.

$\mathrm{HV}$ means the volume of region in objective space enclosed by the nondominated solution set. The larger value of $\mathrm{HV}$ means the better overall performance of algorithm. The HV assessment metrics is defined as

$$
\operatorname{HV}\left(p, z^{r}\right)=\operatorname{VOL}\left(\bigcup_{X \in p}\left[f_{1}(X), z_{1}^{r}\right] \times \cdots\left[f_{\mathrm{m}}(X) z_{\mathrm{m}}^{r}\right]\right),
$$

where $z^{r}=\left(z_{1}^{r}, z_{2}^{r}, \ldots, z_{m}^{r}\right)$ is the point in objective space dominated by any Pareto advantage and $\operatorname{VOL}(\cdot)$ is the Lebesgue measure used to evaluate the volume.

IGD means the average of distances from each reference point to the nearest solution. The smaller value of IGD means the better overall performance of algorithm. The IGD indicator is defined as

$$
\operatorname{IGD}\left(P^{*}, P\right)=\frac{\sum_{X \in p^{*}} d(X, p)}{\left|p^{*}\right|},
$$

where $P$ is the solution set obtained by UF problem, $P^{*}$ is a set composed of Pareto-optimal reference points which are uniform distribution in Pareto front, and $d(X, p)$ is the Euclidean distance between $X$ and any point in $P$.

5.4. Performance Analysis. Each algorithm is run 30 times independently to eliminate contingency. The HV and IGD statistical results of different algorithms evaluated on each problem are shown in Table 3. The Pareto-optimal solutions optimized by $\mathrm{HMOHHO}$ are shown in Figures 5 and 6.

It can be seen from Table 3 that the proposed $\mathrm{HMOHHO}$ algorithm achieves the best results on all the statistical indexes for UF1, UF7, and ZDT1, which means that the proposed $\mathrm{HMOHHO}$ algorithm provides superior diversity and astringency on UF1, UF7, and ZDT1, and the front coverage of Pareto-optimal solutions of $\mathrm{HMOHHO}$ is broader than that of all other algorithms on these test functions.

As to the test functions UF2 and UF4, the proposed HMOHHO algorithm achieves the best HV and IGD results in terms of min, max, and mean values. Although MOHHO obtains the minimum standard deviation of $\mathrm{HV}$ for both UF2 and UF4 problems as well as the minimum standard deviation of IGD for UF4 problem, the standard deviation values of the proposed HMOHHO algorithm are close to the minimum standard deviations. On the whole, $\mathrm{HMOHHO}$ achieves the best convergence average with 
Inputs: Population size $N$, maximum iterations $T$ and archive size $S$

Initialize: Latin hypercube sampling initialize population $X_{i}(i=1,2, \ldots, N)$

Calculate $f_{1}(\cdot)$ and $f_{2}(\cdot)$

Find the non-dominated solutions

while $t<T$

if the archive is full

Crowded area of the archive is deleted by roulette wheel method

Add new solutions to the archive

end if

Select $X_{\text {rabbit }}(t)$ in the archive

for each hawk

if $a b s(E)<1$

$X(t)$ is updated by (14)

else

$X(t)$ is updated by (15)-(24)

end if

$X_{r 1}$ is updated by (27)

if $f\left(X_{r 1}\right)$ dominate $f(X(t))$

$X(t)=X_{r 1}$

end if

$X_{r 2}$ is updated by (28)

if $f\left(X_{r 2}\right)$ dominate $f(X(t))$

$X(t)=X_{r 2}$

end if

end for

Calculate $f_{1}(\cdot)$ and $f_{2}(\cdot)$

Find the non-dominated solutions

$$
t=t+1
$$

end while

return archive

Algorithm 1: Pseudocode of HMOHHO algorithm.

TABLE 2: Test function problems.

\begin{tabular}{|c|c|}
\hline Name & Mathematical formulation \\
\hline UF1 & $\begin{array}{c}f_{1}=x_{1}+\left(2 /\left|J_{1}\right|\right) \sum_{j \in J_{1}}\left[x_{j}-\sin \left(6 \pi x_{1}+(j \pi / n)\right)\right]^{2}, f_{2}=1-\sqrt{x}+\left(2 /\left|J_{2}\right|\right) \sum_{j \in J_{2}}\left[x_{j}-\sin \left(6 \pi x_{1}+(j \pi / n)\right)\right]^{2} \\
J_{1}=\{j \mid \\
j \text { is odd and } 2 \leq j \leq n\}, J_{2}=\{j \mid j \text { is even and } 2 \leq j \leq n\}\end{array}$ \\
\hline UF2 & $\begin{aligned} f_{1} & =x_{1}+\left(2 /\left|J_{1}\right|\right) \sum_{j \in J_{1}} y_{i}^{2}, f_{2}=1-\sqrt{x}+\left(2 /\left|J_{2}\right|\right) \sum_{j \in J_{2}} y_{i}^{2} \\
J_{1} & =\{j \mid j \text { is odd and } 2 \leq j \leq n\}, J_{2}=\{j \mid j \text { is even and } 2 \leq j \leq n\} \\
y_{i} & =\left\{\begin{array}{l}x_{j}-\left[0.3 x_{1}^{2} \cos \left(24 \pi x_{1}+(4 j \pi / n)\right)+0.6 x_{1}\right] \cos \left(6 \pi x_{1}+(j \pi / n)\right) \text { if } j \in J_{1} \\
x_{j}-\left[0.3 x_{1}^{2} \cos \left(24 \pi x_{1}+(4 j \pi / n)\right)+0.6 x_{1}\right] \cos \left(6 \pi x_{1}+(j \pi / n)\right) \text { if } j \in J_{2}\end{array}\right.\end{aligned}$ \\
\hline UF4 & $\begin{array}{l}f_{1}=x_{1}+\left(2 /\left|J_{1}\right|\right) \sum_{j \in J_{1}} h\left(y_{j}\right), f_{2}=1-x_{2}+\left(2 /\left|J_{2}\right|\right) \sum_{j \in J_{2}} h\left(y_{j}\right) \\
J_{1} \text { and } J_{2} \text { are identical to those of UF1, } y_{j}=x_{j}-\sin \left(6 \pi x_{1}+(j \pi / n)\right), j=2,3, \ldots, n, h(t)=\left(|t| /\left(1+e^{2|t|}\right)\right)\end{array}$ \\
\hline UF7 & $\begin{array}{l}f_{1}=\sqrt[5]{x_{1}}+\left(2 /\left|J_{1}\right|\right) \sum_{j \in J_{1}} y_{j}^{2}, f_{2}=\sqrt[5]{x_{1}}+\left(2 /\left|J_{2}\right|\right) \sum_{j \in J_{2}} y_{j}^{2} \\
J_{1} \text { and } J_{2} \text { are identical to those of UF1, } y_{i}=x_{j}-\sin \left(6 \pi x_{1}+(j \pi / n)\right), j=2,3, \ldots, n\end{array}$ \\
\hline ZDT1 & $\begin{array}{l}f_{1}(X)=x_{1} \\
f_{2}(X)=g *\left(1-\sqrt{f_{1} / g}\right) \\
g(X)=1+9 * \sum_{i=2}^{n} x_{i} /(n-1)\end{array}$ \\
\hline ZDT3 & $\begin{array}{l}f_{1}(X)=x_{1} \\
f_{2}(X)=g *\left(1-\sqrt{f_{1} / g}\right)-\left(f_{1} / g\right) \sin \left(10 \pi f_{1}\right) \\
g(X)=1+9 * \sum_{i=2}^{n} x_{i} /(n-1)\end{array}$ \\
\hline ZDT6 & $\begin{array}{l}f_{1}(X)=1-\exp \left(-4 x_{1}\right) \sin ^{6}\left(6 \pi x_{1}\right) \\
f_{2}(X)=g *\left(1-\left(\sqrt{f_{1} / g}\right)^{2}\right) \\
g(X)=1+9 *\left[\sum_{i=2}^{n} x_{i} /(n-1)\right]^{0.25}\end{array}$ \\
\hline
\end{tabular}

The UF test functions search space is $[0,1] \times[-1,1]^{\mathrm{dim}-1}$ and the ZDT test functions search space is $[0,1]^{\mathrm{dim}}$. 
TABle 3: HV and IGD statistical results of different algorithms.

\begin{tabular}{|c|c|c|c|c|c|c|c|c|}
\hline \multirow{2}{*}{ Algorithm } & \multicolumn{4}{|c|}{$\mathrm{HV}$} & \multicolumn{4}{|c|}{ IGD } \\
\hline & Min & Max & Mean & Std & Min & $\operatorname{Max}$ & Mean & Std \\
\hline \multicolumn{9}{|c|}{ UF1 problem (2 objs) } \\
\hline NSGAIIII & 0.1940 & 0.4290 & 0.3642 & 0.6180 & 0.3219 & 0.7399 & 0.4356 & 0.1137 \\
\hline MOPSO & 0.4778 & 0.5843 & 0.5465 & 0.0274 & 0.0818 & 0.1660 & 0.1115 & 0.0197 \\
\hline MOGWO & 0.3580 & 0.5984 & 0.5555 & 0.0441 & 0.0915 & 0.2577 & 0.1191 & 0.0280 \\
\hline МOHНO & 0.5263 & 0.5776 & 0.5263 & 0.0325 & 0.0947 & 0.1816 & 0.1298 & 0.0219 \\
\hline НMOHНO & 0.6198 & 0.6440 & 0.6327 & 0.0058 & 0.0533 & 0.0735 & 0.0619 & 0.0050 \\
\hline \multicolumn{9}{|c|}{ UF2 problem (2 objs) } \\
\hline NSGAIII & 0.3940 & 0.5371 & 0.4710 & 0.0363 & 0.1897 & 0.5096 & 0.3599 & 0.0791 \\
\hline MOPSO & 0.6261 & 0.6626 & 0.6485 & 0.0087 & 0.0479 & 0.1279 & 0.0626 & 0.0147 \\
\hline MOGWO & 0.6170 & 0.6577 & 0.6425 & 0.0101 & 0.0505 & 0.0863 & 0.0652 & 0.0084 \\
\hline МOHНO & 0.6182 & 0.6369 & 0.6259 & 0.0046 & 0.0696 & 0.0876 & 0.0811 & 0.0048 \\
\hline НМОННО & 0.6482 & 0.6721 & 0.6580 & 0.0052 & 0.0412 & 0.0598 & 0.0528 & 0.0041 \\
\hline \multicolumn{9}{|c|}{ UF4 problem (2 objs) } \\
\hline NSGAIII & 0.0649 & 0.3020 & 0.2190 & 0.0592 & 0.0969 & 0.5974 & 0.2359 & 0.1008 \\
\hline MOPSO & 0.2111 & 0.2554 & 0.2263 & 0.0095 & 0.1323 & 0.1778 & 0.1617 & 0.0095 \\
\hline MOGWO & 0.3110 & 0.3593 & 0.3560 & 0.0040 & 0.0580 & 0.0791 & 0.0623 & 0.0054 \\
\hline MOHHO & 0.3440 & 0.3565 & 0.3514 & 0.0038 & 0.0595 & 0.0706 & 0.0632 & 0.0038 \\
\hline НMOHНO & 0.3465 & 0.3623 & 0.3569 & 0.0048 & 0.0564 & 0.0682 & 0.0608 & 0.0042 \\
\hline \multicolumn{9}{|c|}{ UF7 problem (2 objs) } \\
\hline NSGAIII & 0.1170 & 0.3590 & 0.2676 & 0.5870 & 0.2584 & 0.6633 & 0.4056 & 0.0999 \\
\hline MOPSO & 0.1101 & 0.4943 & 0.4088 & 0.1038 & 0.0618 & 0.6739 & 0.1660 & 0.1541 \\
\hline MOGWO & 0.2960 & 0.5050 & 0.4807 & 0.0357 & 0.0585 & 0.3393 & 0.0791 & 0.0495 \\
\hline MOHHO & 0.3765 & 0.4840 & 0.4366 & 0.0269 & 0.0638 & 0.1458 & 0.0994 & 0.0204 \\
\hline HMOHHO & 0.5094 & 0.5359 & 0.5264 & 0.0056 & 0.0337 & 0.0505 & 0.0394 & 0.0038 \\
\hline \multicolumn{9}{|c|}{ ZDT1 problem (2 objs) } \\
\hline NSGAIII & 0.3070 & 0.5069 & 0.4460 & 0.0494 & 0.1893 & 0.4766 & 0.2658 & 0.0729 \\
\hline MOPSO & 0.0628 & 0.6667 & 0.4022 & 0.1686 & 0.0427 & 0.7223 & 0.2907 & 0.1837 \\
\hline MOGWO & 0.6980 & 0.7136 & 0.7078 & 0.0032 & 0.0084 & 0.0271 & 0.0147 & 0.0037 \\
\hline MOHHO & 0.7105 & 0.7153 & 0.7129 & 0.0011 & 0.0088 & 0.0129 & 0.0108 & 0.0010 \\
\hline НMOHНО & 0.7130 & 0.7180 & 0.7162 & 0.0010 & 0.0061 & 0.0101 & $\mathbf{0 . 0 0 7 7}$ & $9.19 E-04$ \\
\hline \multicolumn{9}{|c|}{ ZDT3 problem (2 objs) } \\
\hline NSGAIII & 0.4050 & 0.6339 & 0.5370 & 0.0591 & 0.2059 & 0.3766 & 0.2685 & 0.0468 \\
\hline MOPSO & 0.0157 & 0.7756 & 0.3020 & 0.1844 & 0.1359 & 0.9377 & 0.5330 & 0.1860 \\
\hline MOGWO & 0.5660 & 0.5990 & 0.5810 & 0.0063 & 0.0086 & 0.0249 & 0.0135 & 0.0037 \\
\hline MOHHO & 0.5777 & 0.5906 & 0.5809 & 0.0022 & 0.0081 & 0.0130 & 0.0106 & 0.0013 \\
\hline НМОННО & 0.5797 & 0.5897 & 0.5821 & 0.0018 & 0.0070 & 0.0099 & 0.0085 & $9.73 E-05$ \\
\hline \multicolumn{9}{|c|}{ ZDT6 problem (2 objs) } \\
\hline NSGAIII & 0.1670 & 0.3833 & 0.3510 & 0.0508 & 0.0030 & 0.0767 & 0.0123 & 0.0153 \\
\hline MOPSO & 0.3135 & 0.3862 & 0.3771 & 0.0213 & 0.0026 & 0.0208 & 0.0057 & 0.0048 \\
\hline MOGWO & 0.3650 & 0.3857 & 0.3798 & 0.0058 & 0.0033 & 0.1036 & 0.0246 & 0.0284 \\
\hline МOHНO & 0.3816 & 0.3853 & 0.3837 & 0.0008 & 0.0045 & 0.0215 & 0.0065 & 0.0030 \\
\hline НМОННО & 0.3846 & 0.3866 & 0.3857 & $5.75 E-04$ & 0.0029 & 0.0065 & 0.0042 & $8.27 E-04$ \\
\hline
\end{tabular}

well stability. It also can be seen that the worst results belong to NSGAIII due to the fact that NSGAIII easily falls into local optimums.

As to the test function ZDT3, the proposed $\mathrm{HMOHHO}$ algorithm achieves the best $\mathrm{HV}$ results in terms of min, mean, and std values as well as the best IGD in terms of all four values. Although MOPSO obtains the maximum HV value, its $\mathrm{HV}$ results in terms of mean and std values are not so satisfactory. With regard to the test function ZDT6, the proposed HMOHHO algorithm achieves the best IGD results in terms of max, mean, and std values as well as the best $\mathrm{HV}$ in terms of all four values. Although MOPSO obtains the minimum IGD value, its IGD results in terms of mean and std values are not so satisfactory.

On the whole, based on the above contrastive analysis for Table 3, the application for test functions shows that the performance of the proposed HMOHHO algorithm achieves better convergence and stability than other contrastive algorithms, indicating that the proposed $\mathrm{HMOHHO}$ is able to realize remarkable diversity and astringency ability in settling multiobjective problems.

\section{Case Experiment}

6.1. Comparison of Control Effects between Two Controllers. In this experiment, FOPID and PID controllers of HTGS were applied to obtain dynamic performance under noload and on-load disturbance conditions. Experiment was run over a limited time frame of $20 \mathrm{~s}$. Considering two objective functions $f_{1}(\cdot)$ and $f_{2}(\cdot)$, the Pareto fronts obtained by HMOHHO for HTGS multiobjective control problem based on PID and FOPID controllers are shown 


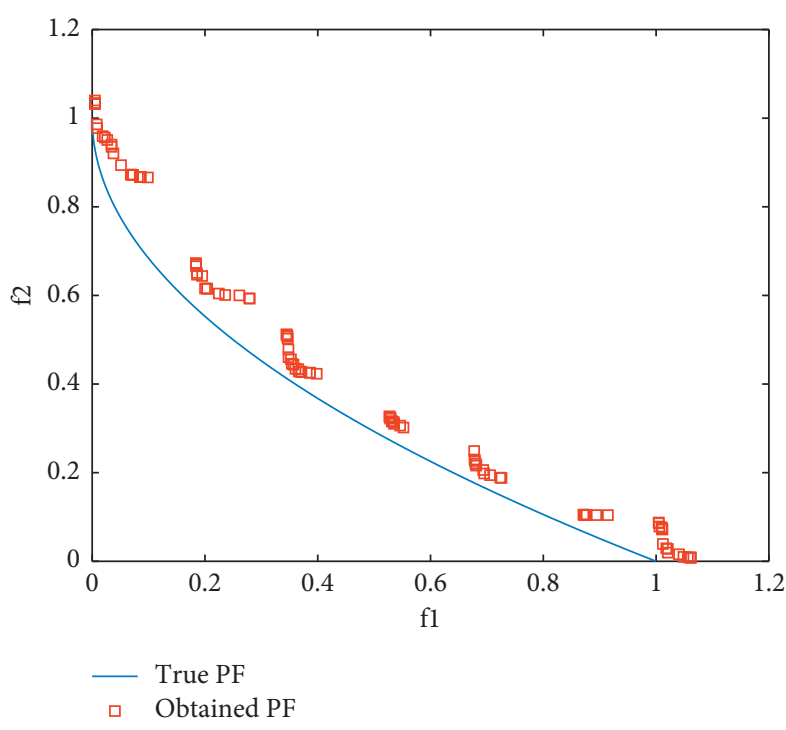

(a)

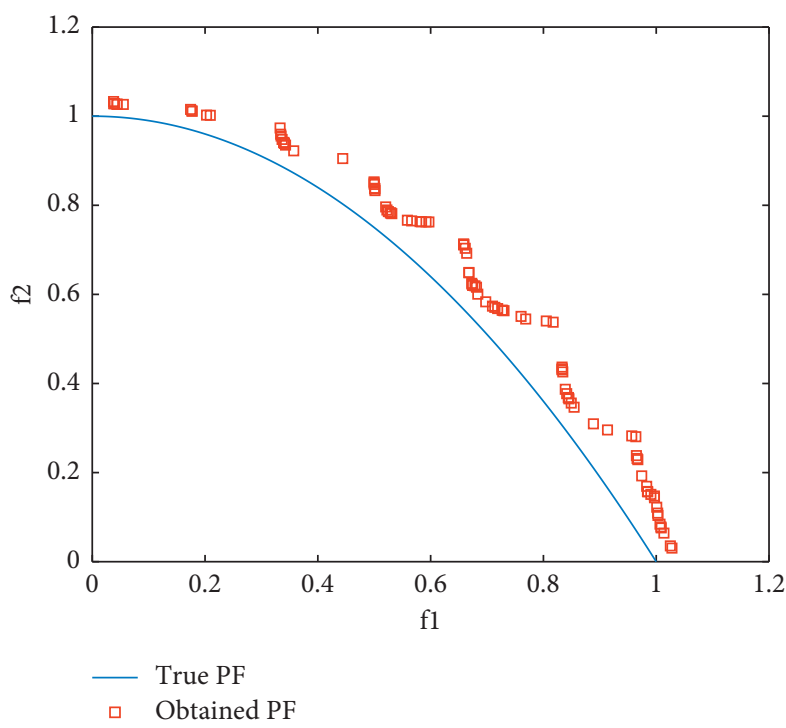

(c)

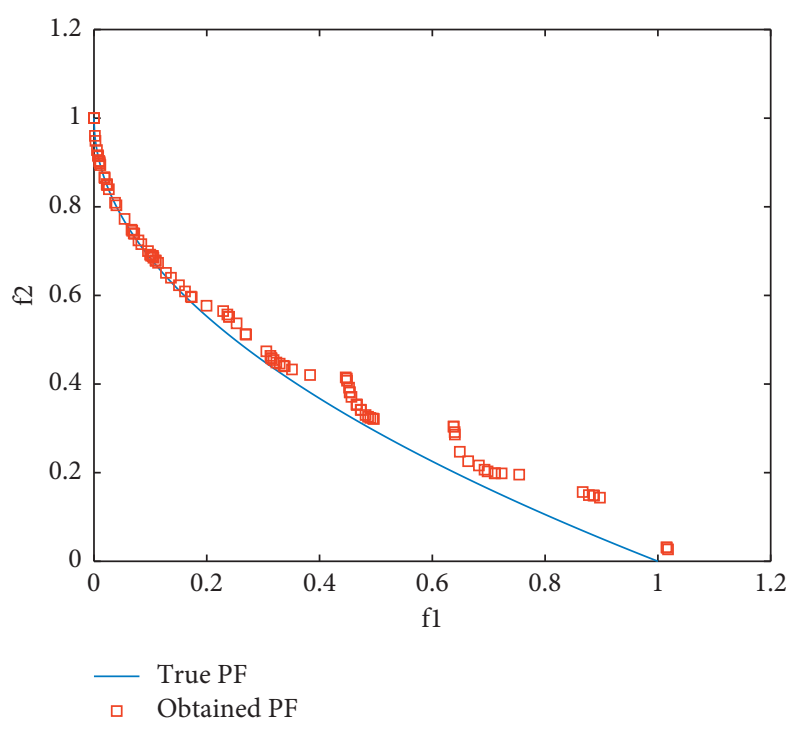

(b)

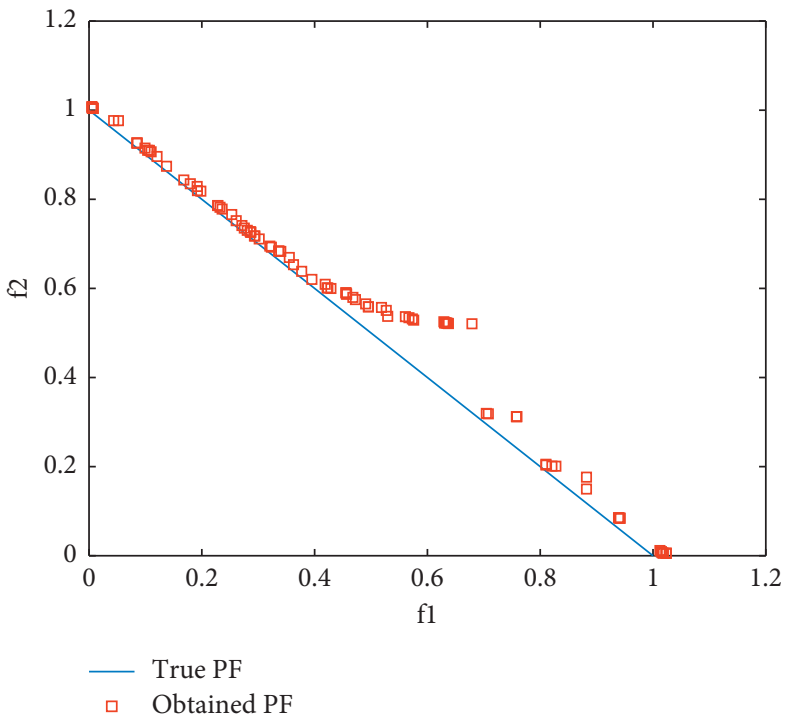

(d)

FIGURE 5: Pareto-optimal solutions obtained by HMOHHO for UF1, UF2, UF4, and UF7. (a) HMOHHO for UF1 problem, (b) HMOHHO for UF2 problem, (c) HMOHHO for UF4 problem, and (d) HMOHHO for UF7 problem.

in Figure 7. As seen in Figure 7, the Pareto front based on FOPID controller is completely located in the below portion of the result based on PID controller, indicating that the values of $f_{1}(\cdot)$ and $f_{2}(\cdot)$ are smaller based on FOPID controller. Thus, better parameters are deduced based on FOPID controller to achieve better dynamic performance for HTGS.

Seven control schemes selected from the Pareto-optimal solution set are shown in Table 4, where the first six ones are from the Pareto front with FOPID controller and the last one is from the Pareto front with PID controller. The results obtained by control schemes 3 and 7 in Table 4 are shown in Figure 8. In the case of no-load disturbance condition, the system with FOPID controller has a less response rise time and low overshoot, while the system with PID controller takes a long time to restore. Under on-load disturbance condition, the system with FOPID controller can recover quickly.

6.2. The Pareto-Optimal Sets with Different Algorithms and Analysis. In this experiment, NSGA-III, MOPSO, MOGWO, MOHHO, and the proposed HMOHHO algorithms were applied to optimize HTGS under no-load and on-load operating conditions. The parameters of HTGS model are listed in Table 1. The simulation time is $20 \mathrm{~s}$. The maximum iteration is 150 and each algorithm is run 10 times independently. The best results for each algorithm in the repeated experiments are shown in Figure 9, from which it can be seen that the solution set of the proposed HMOHHO achieves more diversity than the solutions of other algorithms. 


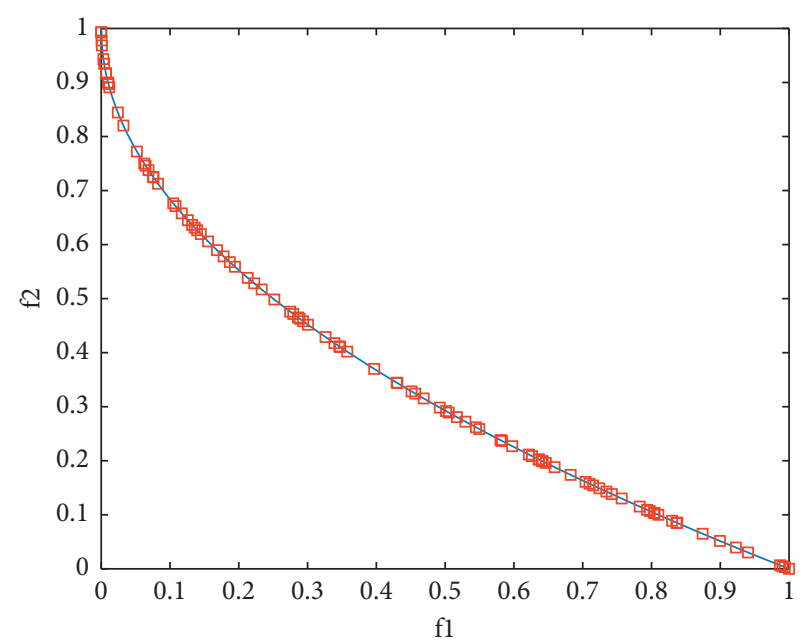

- True PF

$\quad$ Obtained PF

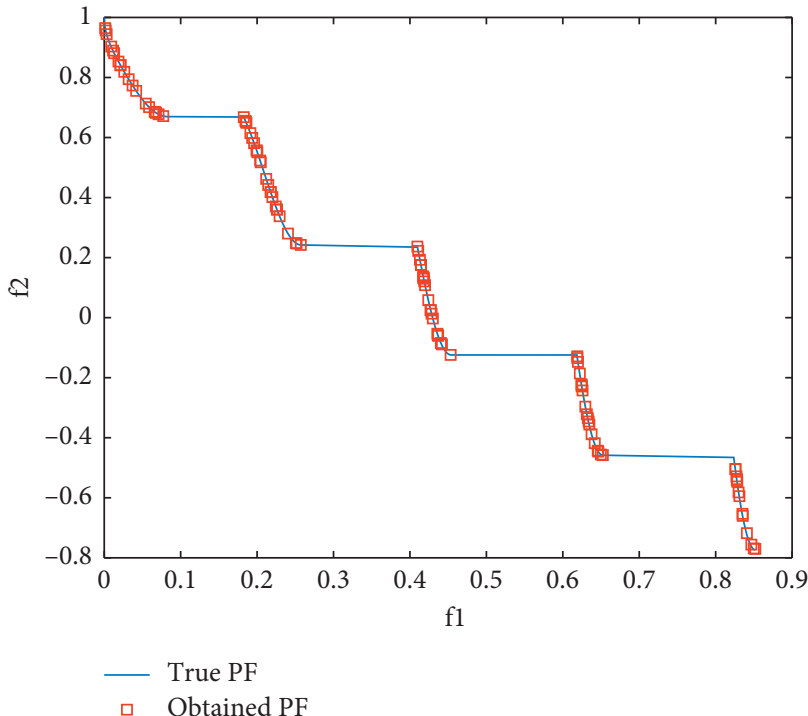

(b)

(a)

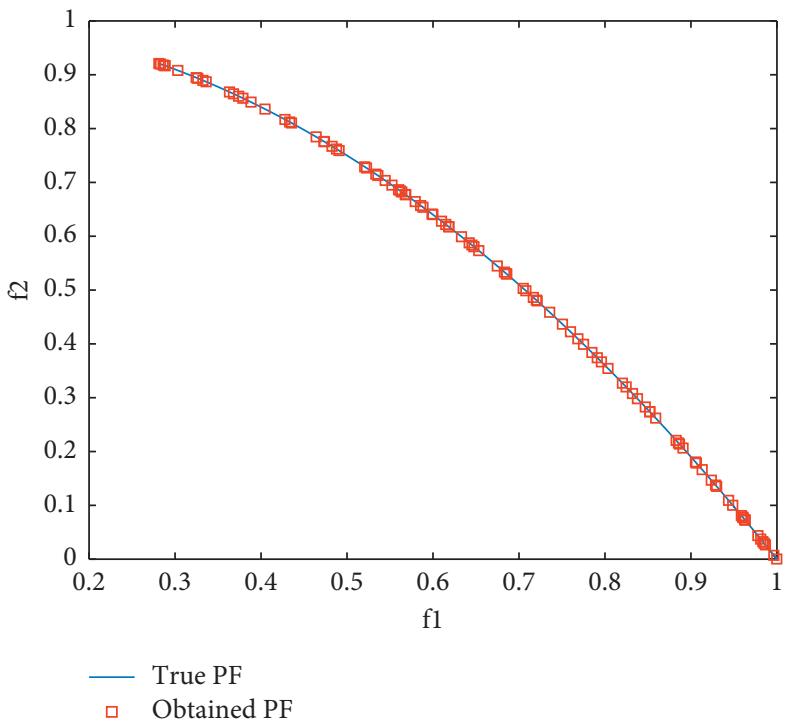

(c)

FIGURE 6: Pareto-optimal solutions obtained by HMOHHO for ZDT1, ZDT3, and ZDT6. (a) HMOHHO for ZDT1 problem, (b) HMOHHO for ZDT3 problem, and (c) HMOHHO for ZDT6 problem.

Three control schemes from the optimal solution set in Pareto front as shown in Table 4 are selected to conduct a comparative experiment of transient process response. The transient process responses with the three control schemes are shown in Figure 10 and the corresponding performance indexes are listed in Table 4. As exhibited in Figure 10, all of the three control schemes realize good control stability under no-load and on-load disturbance conditions. Besides, it can be concluded from Table 4 that the ITAE indexes under no-load and on-load are with a certain contradiction. Specifically, scheme 5 achieves the smallest overshoot among the three schemes under no-load disturbance condition, while its stability is the worst under on-load disturbance condition. On the contrary, scheme 3 is the most stable under on-load disturbance condition, while the overshoot is larger than the other two control schemes under no-load disturbance condition. Scheme 4 is a balanced one which is suitable for the transformation of operating conditions. In order to achieve better control quality of HTGS, the control schemes with good control effect should be chosen by decision makers in the final solution according to the special requirements of HTGS. 


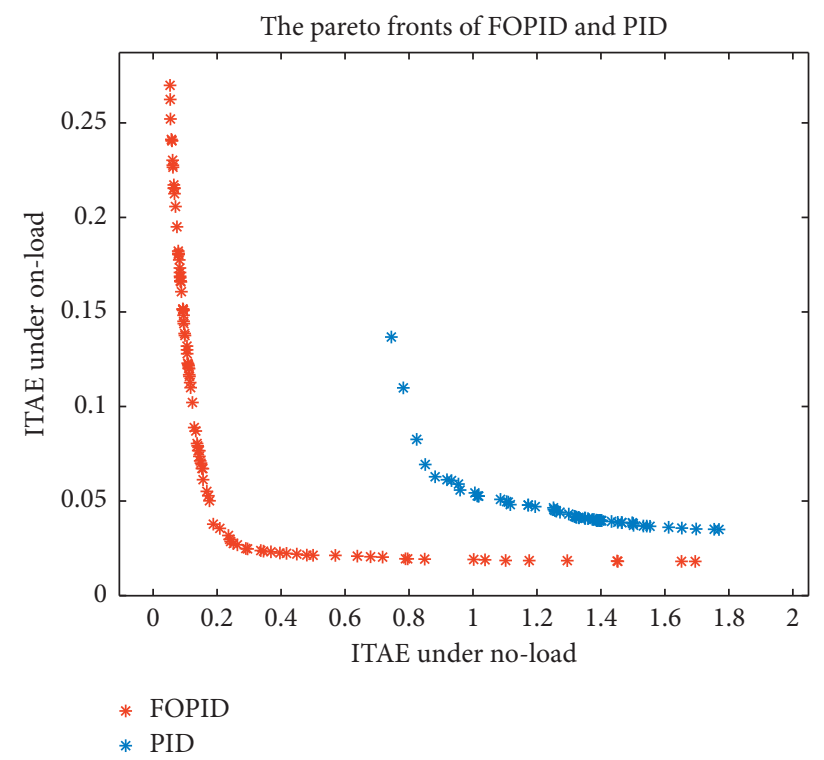

FIGURe 7: Pareto fronts optimized by HMOHHO for multiobjective control problems of HTGS based on PID and FOPID controllers.

TABle 4: Control schemes selected from the Pareto-optimal solution set.

\begin{tabular}{lccccccc}
\hline \multirow{2}{*}{ Control scheme } & \multicolumn{4}{c}{ Parameters of HGTS } & \multicolumn{2}{c}{ Performance indexes } \\
& $K_{\mathrm{p}}$ & $K_{\mathrm{i}}$ & $K_{\mathrm{d}}$ & $\lambda$ & $\mu$ & ITAE under no-load & ITAE under on-load \\
\hline 1 & 5.6540 & 5.4158 & 2.2957 & 0.9941 & 0.3083 & 0.2953 & 0.0247 \\
2 & 5.7211 & 5.0053 & 1.5468 & 0.9916 & 0.2826 & 0.2562 & 0.0265 \\
3 & 5.7143 & 4.8778 & 1.9615 & 0.9906 & 0.2679 & 0.2515 & 0.0270 \\
4 & 5.8988 & 4.8668 & 1.5468 & 0.9916 & 0.2766 & 0.2423 & 0.0286 \\
5 & 5.8163 & 4.7606 & 1.5247 & 0.9891 & 0.2134 & 0.2355 & 0.0318 \\
7 & 6.1125 & 4.1903 & 1.1552 & 0.9894 & 0.2918 & 0.2153 & 0.0330 \\
\hline
\end{tabular}

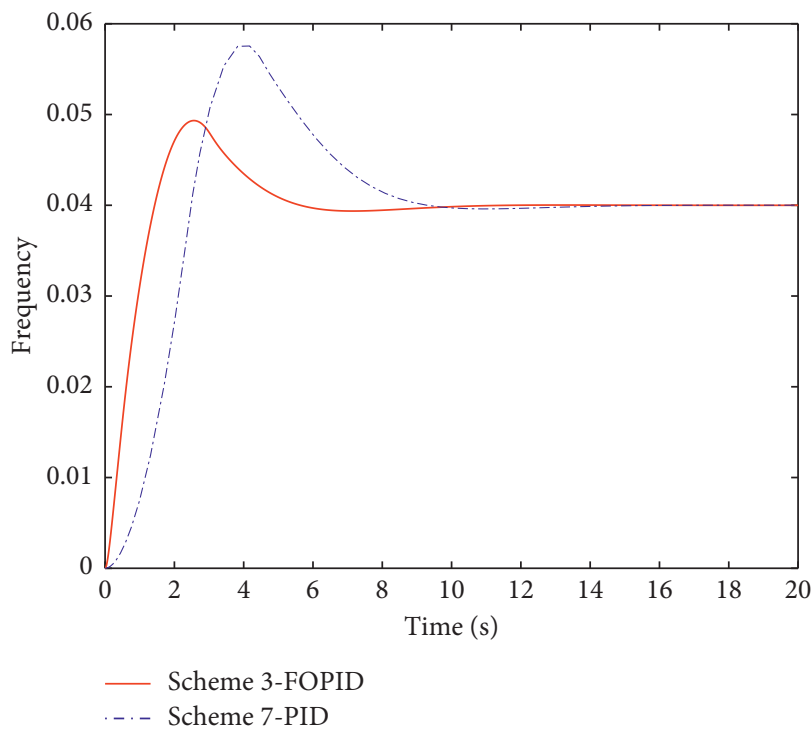

(a)

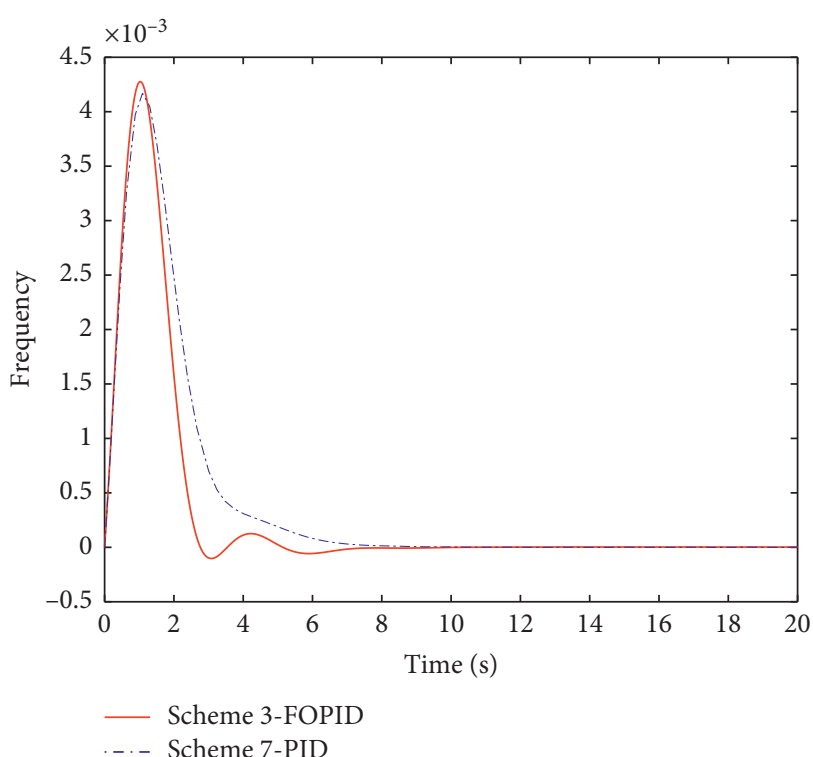

(b)

FIgURE 8: Comparison of PID and FOPID under two operating conditions. (a) No-load operating condition; (b) on-load operating condition. 


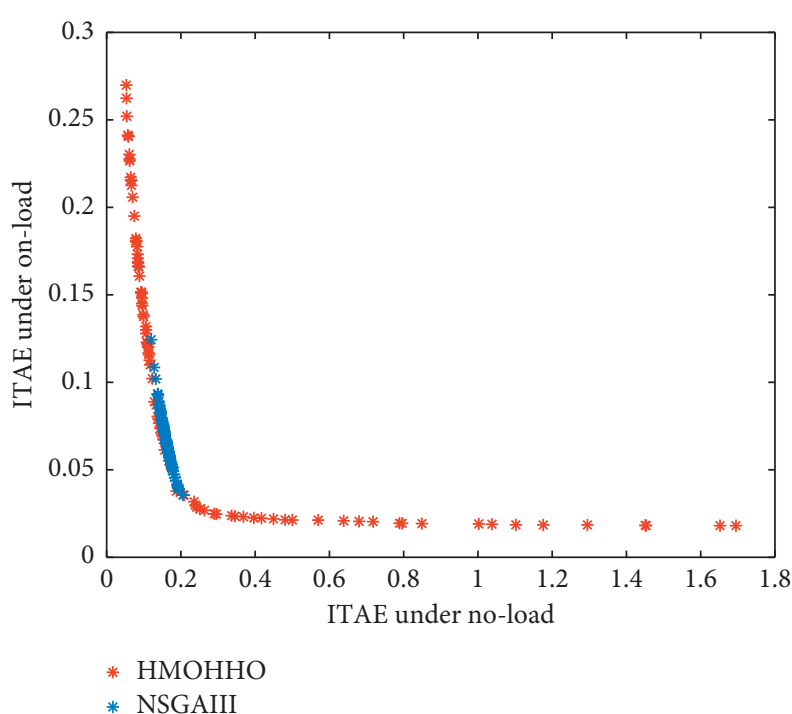

(a)

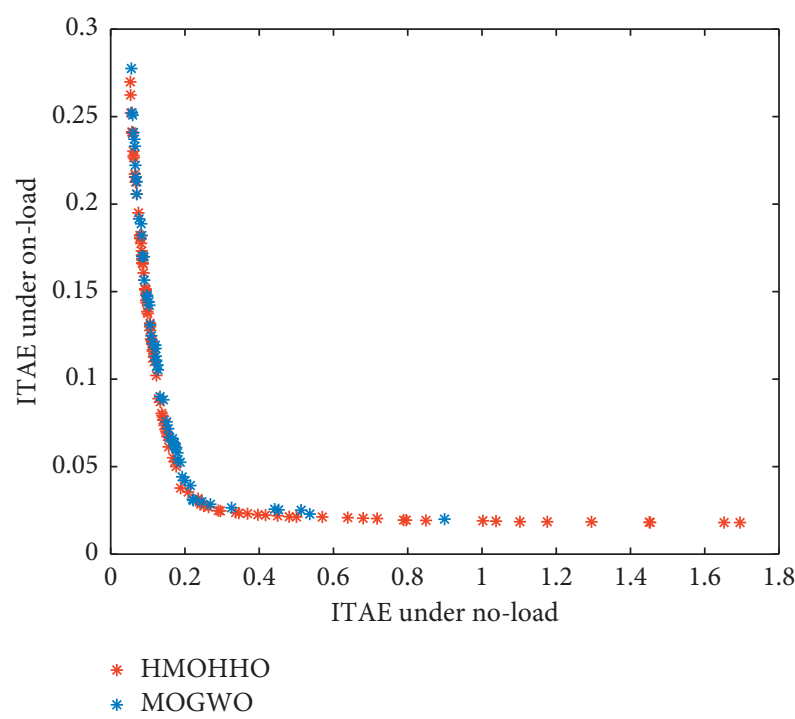

(c)

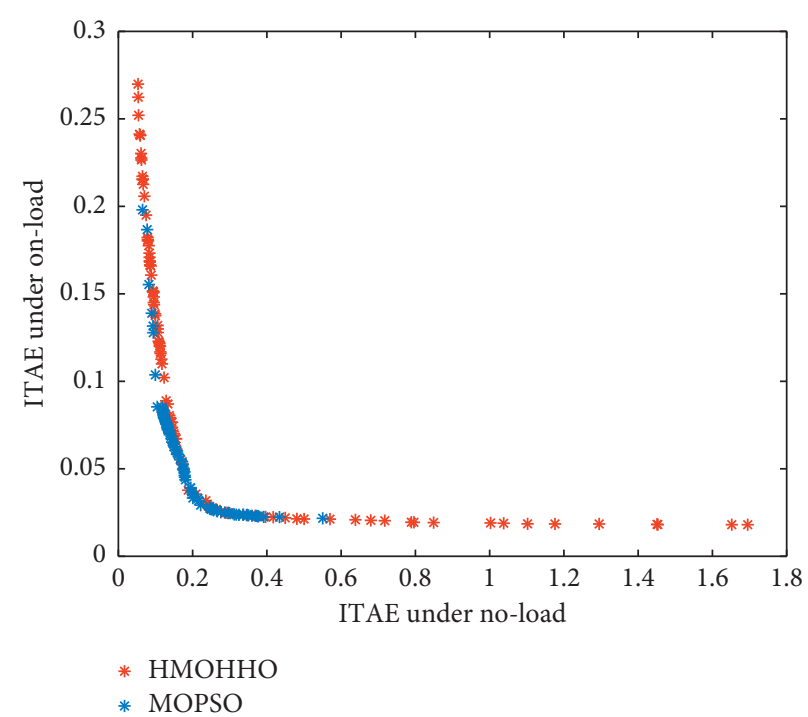

(b)

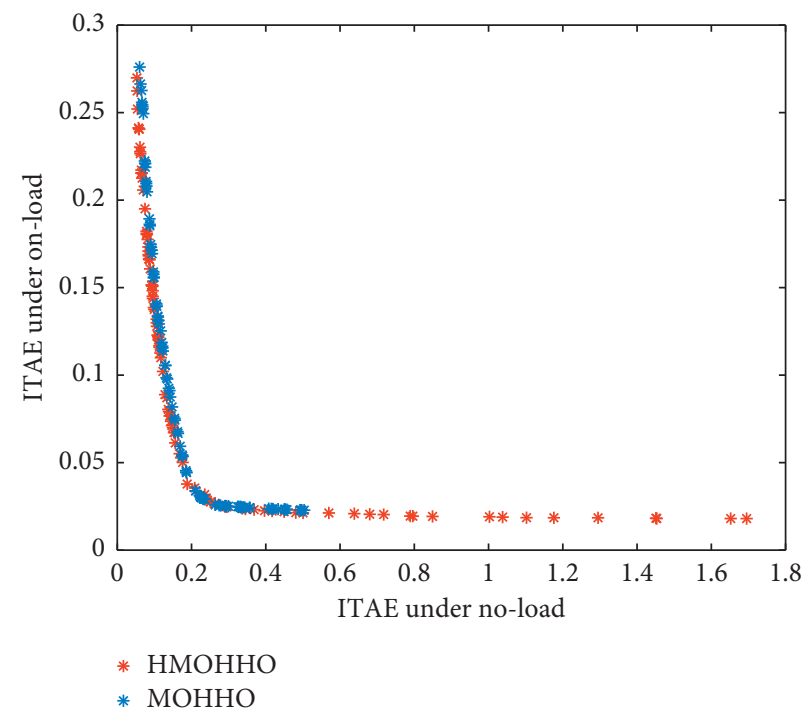

(d)

FIgure 9: The Pareto fronts of different algorithms. (a) The Pareto fronts of HMOHHO and NSGAIII. (b) The Pareto fronts of HMOHHO and MOPSO. (c) The Pareto fronts of HMOHHO and MOGWO. (d) The Pareto fronts of HMOHHO and MOHHO.

\section{Conclusions}

To enhance the controlling parameter tuning applicability of HTGS under changeable operating conditions, the multiobjective optimization problem of FOPID controller is built by considering no-load disturbance and on-load disturbance operation conditions, where the ITAE performance indicators under both operation conditions are employed as the objective functions. Then, the newly proposed Harris hawks optimization is extended to the multiobjective version MOHHO for solving the optimization problem.
Additionally, the global searching capability of $\mathrm{MOHHO}$ is enhanced greatly through coupling $\mathrm{MOHHO}$ with hybrid strategies (HMOHHO) which include Latin hypercube sampling initialization, modified differential evolution operator, and mutation operator as well as replacing the linear model of rabbit energy with a nonlinear one. Subsequently, the proposed $\mathrm{HMOHHO}$ algorithm is tested on several test functions and compared with NSGAIII, MOGWO, MOPSO, and $\mathrm{MOHHO}$, which verifies the effectiveness of the proposed $\mathrm{HMOHHO}$ algorithm. The practical application as well as contrastive analysis shows that the constructed 


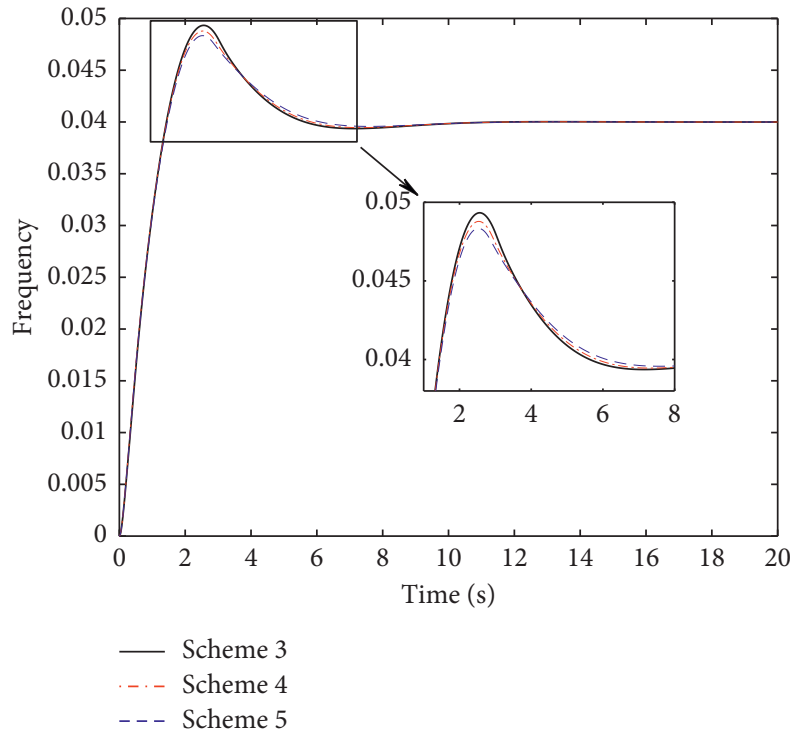

(a)

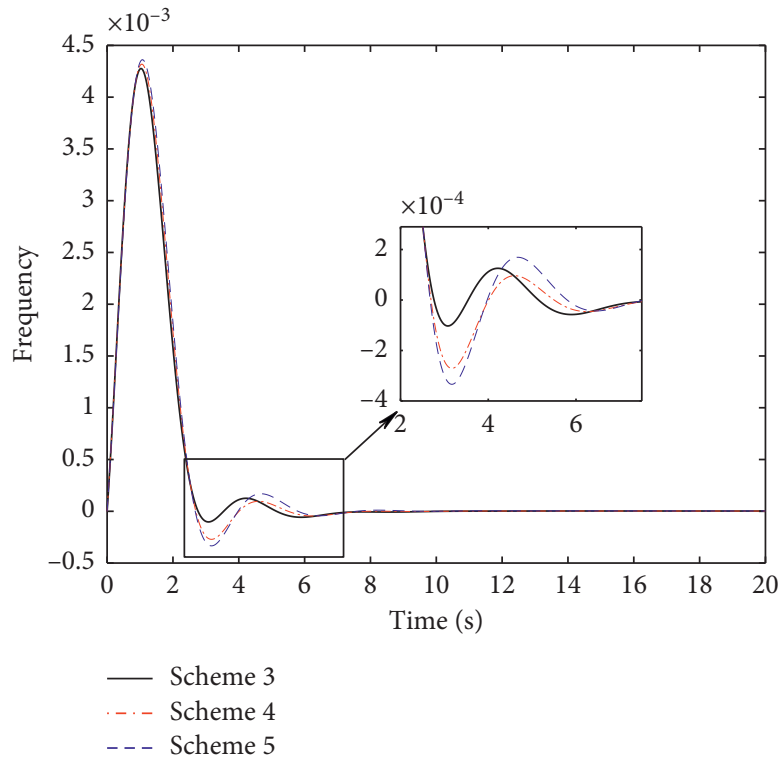

(b)

FIgURE 10: Transient process responses with different control schemes. (a) No-load operating condition; (b) on-load operating condition.

multiobjective problem of FOPID controller is suitable for HTGS under changeable operating conditions and the proposed HMOHHO algorithm possesses outstanding feasibility and superiority among all the algorithms.

\section{Data Availability}

The data used to support the findings of this study are available from the corresponding author upon request.

\section{Conflicts of Interest}

The authors declare no conflicts of interest.

\section{Acknowledgments}

This work was supported by the National Natural Science Foundation of China under Grant no. 51741907 and the Open Fund of Hubei Provincial Key Laboratory for Operation and Control of Cascaded Hydropower Station under Grant no. 2017KJX06.

\section{References}

[1] Z. Dong, J. Zhao, F. Wen, and Y. Xue, "From smart grid to energy internet: basic concept and research framework," Automation of Electric power Systems, vol. 34, no. 15, pp. 1-11, 2014.

[2] W. Fu, K. Wang, J. Tan, and K. Zhang, "A composite framework coupling multiple feature selection, compound prediction models and novel hybrid swarm optimizer-based synchronization optimization strategy for multi-step ahead short-term wind speed forecasting," Energy Conversion and Management, vol. 205, Article ID 112461, 2020.

[3] Z. Jiang, R. Li, A. Li, and C. Ji, "Runoff forecast uncertainty considered load adjustment model of cascade hydropower stations and its application," Energy, vol. 158, pp. 693-708, 2018.

[4] Z. Zhao, J. Yang, W. Yang, J. Hu, and M. Chen, "A coordinated optimization framework for flexible operation of pumped storage hydropower system: nonlinear modeling, strategy optimization and decision making," Energy Conversion and Management, vol. 194, pp. 75-93, 2019.

[5] W. Guo and J. Yang, "Stability performance for primary frequency regulation of hydro-turbine governing system with surge tank," Applied Mathematical Modelling, vol. 54, pp. 446-466, 2018.

[6] F. Zhang, D. Appiah, F. Hong et al., "Energy loss evaluation in a side channel pump under different wrapping angles using entropy production method," International Communications in Heat and Mass Transfer, vol. 113, p. 104526, 2020.

[7] W. Fu, K. Wang, C. Li, X. Li, Y. Li, and H. Zhong, "Vibration trend measurement for a hydropower generator based on optimal variational mode decomposition and an LSSVM improved with chaotic sine cosine algorithm optimization," Measurement Science and Technology, vol. 30, no. 1, Article ID 015012, 2019.

[8] W. Fu, K. Wang, C. Zhang, and J. Tan, "A hybrid approach for measuring the vibrational trend of hydroelectric unit with enhanced multi-scale chaotic series analysis and optimized least squares support vector machine," Transactions of the Institute of Measurement and Control, vol. 41, no. 15, pp. 4436-4449, 2019.

[9] W. Guo and Z. Peng, "Hydropower system operation stability considering the coupling effect of water potential energy in surge tank and power grid," Renewable Energy, vol. 134, pp. 846-861, 2019.

[10] X. Yuan, Z. Chen, Y. Yuan, and Y. Huang, "Design of fuzzy sliding mode controller for hydraulic turbine regulating system via input state feedback linearization method," Energy, vol. 93, pp. 173-187, 2015.

[11] S. Mobayen, "Design of LMI-based sliding mode controller with an exponential policy for a class of underactuated systems," Complexity, vol. 21, no. 5, pp. 117-124, 2016. 
[12] S. Mobayen, "Chaos synchronization of uncertain chaotic systems using composite nonlinear feedback based integral sliding mode control," ISA Transactions, vol. 77, pp. 100-111, 2018.

[13] S. Mobayen, "A novel global sliding mode control based on exponential reaching law for a class of underactuated systems with external disturbances," Journal of Computational and Nonlinear Dynamics, vol. 11, no. 2, Article ID 021011, 2016.

[14] S. Huang, B. Zhou, S. Bu et al., "Robust fixed-time sliding mode control for fractional-order nonlinear hydro-turbine governing system," Renewable Energy, vol. 139, pp. 447-458, 2019.

[15] Y. Yi and D. Chen, "Disturbance observer-based backstepping sliding mode fault-tolerant control for the hydro-turbine governing system with dead-zone input," ISA Transactions, vol. 88, pp. 127-141, 2019.

[16] Y. Tian, B. Wang, D. Zhu, and F. Wu, "Takagi-Sugeno fuzzy generalised predictive control of a time-delay non-linear hydro-turbine governing system," Iet Renewable Power Generation, vol. 13, no. 13, pp. 2338-2345, 2019.

[17] C. Li, Y. Mao, J. Zhou, N. Zhang, and X. An, "Design of a fuzzy-PID controller for a nonlinear hydraulic turbine governing system by using a novel gravitational search algorithm based on Cauchy mutation and mass weighting," Applied Soft Computing, vol. 52, pp. 290-305, 2017.

[18] C. Jiang, Y. Ma, and C. Wang, "PID controller parameters optimization of hydro-turbine governing systems using deterministic-chaotic-mutation evolutionary programming (DCMEP)," Energy Conversion and Management, vol. 47, no. 9-10, pp. 1222-1230, 2006.

[19] A. Khodabakhshian and R. Hooshmand, "A new PID controller design for automatic generation control of hydro power systems," International Journal of Electrical Power \& Energy Systems, vol. 32, no. 5, pp. 375-382, 2010.

[20] S. Sondhi and Y. V. Hote, "Fractional order PID controller for load frequency control," Energy Conversion and Management, vol. 85, pp. 343-353, 2014.

[21] X. Wu, Y. Xu, J. Liu, C. Lv, J. Zhou, and Q. Zhang, "Characteristics analysis and fuzzy fractional-order PID parameter optimization for primary frequency modulation of a pumped storage unit based on a multi-objective gravitational search algorithm," Energies, vol. 13, no. 1, p. 137, 2019.

[22] Y. Xu, J. Zhou, Y. Zhang, W. Fu, Y. Zheng, and X. Zhang, "Parameter optimization of robust non-fragile fractional order PID controller for pump turbine governing system," in Proceedings of the 2016 Sixth International Conference on Instrumentation \& Measurement, Computer, Communication and Control (IMCCC), pp. 15-18, IEEE, Harbin, China, July 2016.

[23] C. Li, N. Zhang, X. Lai, J. Zhou, and Y. Xu, "Design of a fractional-order PID controller for a pumped storage unit using a gravitational search algorithm based on the Cauchy and Gaussian mutation," Information Sciences, vol. 396, pp. 162-181, 2017.

[24] C. Xu and D. Qian, "Governor design for a hydropower plant with an upstream surge tank by GA-based fuzzy reducedorder sliding mode," Energies, vol. 8, no. 12, pp. 13442-13457, 2015.

[25] A. Panwar, G. Sharma, I. Nasiruddin, and R. C. Bansal, "Frequency stabilization of hydro-hydro power system using hybrid bacteria foraging PSO with UPFC and HAE," Electric Power Systems Research, vol. 161, pp. 74-85, 2018.

[26] H. Fang, L. Chen, and Z. Shen, "Application of an improved PSO algorithm to optimal tuning of PID gains for water turbine governor," Energy Conversion and Management, vol. 52, no. 4, pp. 1763-1770, 2011.

[27] N. Zhang, C. Li, R. Li, X. Lai, and Y. Zhang, "A mixed-strategy based gravitational search algorithm for parameter identification of hydraulic turbine governing system," KnowledgeBased Systems, vol. 109, pp. 218-237, 2016.

[28] C. Lv, Y. Xu, X. Wu, and Q. Zhang, "Characteristic analysis and optimal regulation of primary frequency regulation condition in low water head area based on hydraulic-mechanical-electrical coupling model of pumped storage unit," Complexity, vol. 2020, Article ID 6161784, 17 pages, 2020.

[29] T. Tian, C. Liu, Q. Guo, Y. Yuan, W. Li, and Q. Yan, “An improved ant lion optimization algorithm and its application in hydraulic turbine governing system parameter identification," Energies, vol. 11, no. 1, p. 95, 2018.

[30] W. Fu, J. Tan, X. Zhang, T. Chen, and K. Wang, "Blind parameter identification of MAR model and mutation hybrid GWO-SCA optimized SVM for fault diagnosis of rotating machinery," Complexity, vol. 2019, Article ID 3264969, 17 pages, 2019.

[31] W. Fu, K. Shao, J. Tan, and K. Wang, "Fault diagnosis for rolling bearings based on composite multiscale fine-sorted dispersion entropy and SVM with hybrid mutation SCAHHO algorithm optimization," IEEE Access, vol. 8, pp. 13086-13104, 2020.

[32] W. Fu, K. Wang, C. Li, and J. Tan, "Multi-step short-term wind speed forecasting approach based on multi-scale dominant ingredient chaotic analysis, improved hybrid GWO-SCA optimization and ELM," Energy Conversion and Management, vol. 187, pp. 356-377, 2019.

[33] W. Fu, K. Wang, J. Zhou, Y. Xu, J. Tan, and T. Chen, “A hybrid approach for multi-step wind speed forecasting based on multi-scale dominant ingredient chaotic analysis, KELM and synchronous optimization strategy," Sustainability, vol. 11, no. 6, p. 1804, 2019.

[34] W. Fu, K. Wang, J. Tan, and K. Shao, "Vibration tendency prediction approach for hydropower generator fused with multiscale dominant ingredient chaotic analysis, adaptive mutation grey wolf optimizer, and KELM," Complexity, vol. 2020, Article ID 4516132, 20 pages, 2020.

[35] Z. Chen, X. Yuan, B. Ji, P. Wang, and H. Tian, "Design of a fractional order PID controller for hydraulic turbine regulating system using chaotic non-dominated sorting genetic algorithm II," Energy Conversion and Management, vol. 84, pp. 390-404, 2014.

[36] T. Piraisoodi, W. I. M. Siluvairaj, and M. A. K. Kappuva, "Multi-objective robust fuzzy fractional order proportional-integral-derivative controller design for nonlinear hydraulic turbine governing system using evolutionary computation techniques," Expert Systems, vol. 36, no. 2, 2019.

[37] X. Lai, C. Li, J. Zhou, and N. Zhang, "Multi-objective optimization of the closure law of guide vanes for pumped storage units," Renewable Energy, vol. 139, pp. 302-312, 2019.

[38] C. Zhang, T. Peng, C. Li, W. Fu, X. Xia, and X. Xue, "Multiobjective optimization of a fractional-order PID controller for pumped turbine governing system using an improved NSGA-III algorithm under multiworking conditions," Complexity, vol. 2019, Article ID 5826873, 18 pages, 2019.

[39] X. Xia, J. Ji, C.-s. Li, X. Xue, X. Wang, and C. Zhang, "Multiobjective optimal control for hydraulic turbine governing system based on an improved MOGWO algorithm," Complexity, vol. 2019, Article ID 3745924, 14 pages, 2019.

[40] X. Bi and C. Wang, "An improved NSGA-III algorithm based on objective space decomposition for many-objective 
optimization," Soft Computing, vol. 21, no. 15, pp. 4269-4296, 2017.

[41] C. A. C. Coello and M. S. Lechuga, "MOPSO: a proposal for multiple objective particle swarm optimization," in Proceedings of the 2002 Congress on Evolutionary Computation CEC'02 (Cat. No. 02TH8600), pp. 1051-1056, Honolulu, HI, USA, May 2002.

[42] S. Mirjalili, S. Saremi, S. M. Mirjalili, and L. d. S. Coelho, "Multi-objective grey wolf optimizer: a novel algorithm for multi-criterion optimization," Expert Systems with Applications, vol. 47, pp. 106-119, 2016.

[43] A. A. Heidari, S. Mirjalili, H. Faris, I. Aljarah, M. Mafarja, and H. Chen, "Harris hawks optimization: algorithm and applications," Future Generation Computer Systems, vol. 97, pp. 849-872, 2019.

[44] J. T. Machado, V. Kiryakova, and F. Mainardi, "Recent history of fractional calculus," Communications in Nonlinear Science and Numerical Simulation, vol. 16, no. 3, pp. 1140-1153, 2011.

[45] L. Liu, F. Pan, and D. Xue, "Variable-order fuzzy fractional PID controller," ISA Transactions, vol. 55, pp. 227-233, 2015.

[46] I. Pan and S. Das, "Chaotic multi-objective optimization based design of fractional order $\mathrm{PI} \lambda \mathrm{D} \mu$ controller in AVR system," International Journal of Electrical Power \& Energy Systems, vol. 43, no. 1, pp. 393-407, 2012.

[47] D. Xue, Fractional-Order Control Systems: Fundamentals and Numerical Implementations, Walter de Gruyter GmbH \& Co KG, Berlin, Germany, 2017.

[48] Z. Chen, X. Yuan, H. Tian, and B. Ji, "Improved gravitational search algorithm for parameter identification of water turbine regulation system," Energy Conversion and Management, vol. 78, pp. 306-315, 2014.

[49] D. Liu, Z. Xiao, H. Li, D. Liu, X. Hu, and O. P. Malik, "Accurate parameter estimation of a hydro-turbine regulation system using adaptive fuzzy particle swarm optimization," Energies, vol. 12, no. 20, p. 3903, 2019.

[50] G. Karer and I. Škrjanc, "Interval-model-based global optimization framework for robust stability and performance of PID controllers," Applied Soft Computing, vol. 40, pp. 526543, 2016.

[51] Y. Liu, H. Qin, Z. Zhang et al., "A region search evolutionary algorithm for many-objective optimization," Information Sciences, vol. 488, pp. 19-40, 2019.

[52] L. Li, Q. Lin, S. Liu, D. Gong, C. A. Coello Coello, and Z. Ming, "A novel multi-objective immune algorithm with a decomposition-based clonal selection," Applied Soft Computing, vol. 81, Article ID 105490, 2019.

[53] V. Stanovov, S. Akhmedova, and E. Semenkin, "Selective pressure strategy in differential evolution: exploitation improvement in solving global optimization problems," Swarm and Evolutionary Computation, vol. 50, Article ID 100463, 2019.

[54] Z. Jiang, P. Liu, C. Ji, H. Zhang, and Y. Chen, "Ecological flow considered multi-objective storage energy operation chart optimization of large-scale mixed reservoirs," Journal of Hydrology, vol. 577, Article ID 123949, 2019.

[55] S. Poles, Y. Fu, and E. Rigoni, The Effect of Initial Population Sampling on the Convergence of Multi-Objective Genetic Algorithms, pp. 123-133, Springer Berlin Heidelberg, Berlin, Heidelberg, 2009.

[56] P. A. Grudniewski and A. J. Sobey, "Multi-level selection genetic algorithm applied to CEC'09 test instances," in Proceedings of the 2017 IEEE Congress on Evolutionary Computation (CEC), pp. 1613-1620, IEEE, San Sebastian, Spain, June 2017.
[57] E. Zitzler, K. Deb, and L. Thiele, "Comparison of multiobjective evolutionary algorithms: empirical results," Evolutionary Computation, vol. 8, no. 2, pp. 173-195, 2000.

[58] S. Mirjalili, P. Jangir, S. Z. Mirjalili, S. Saremi, and I. N. Trivedi, "Optimization of problems with multiple objectives using the multi-verse optimization algorithm," Knowledge-Based Systems, vol. 134, pp. 50-71, 2017. 